\title{
Strategic Decision-Making in a Global Context: The Comprehension Effect of Foreign Language Use on Cooperation
}

\author{
Diemo Urbig ${ }^{1,2} \cdot$ Katrin Muehlfeld ${ }^{3,4} \cdot$ Vivien D. Procher ${ }^{5,6}$ (D) \\ Arjen van Witteloostuijn ${ }^{7,8}$
}

Received: 7 July 2019 / Revised: 2 February 2020 / Accepted: 17 February 2020 / Published online: 3 April 2020 (c) The Author(s) 2020

\begin{abstract}
With increasing globalization comes an increasing number of people communicating in foreign languages when making strategic decisions. We develop a theoretical model in which comprehension constitutes an essential mediator for the effects of using a foreign language on cooperation in global business contexts. To resolve conceptual ambiguities, we separate information processing leading to comprehension from decision-making employing the previously comprehended information. For the first step, we demonstrate how using a foreign language can, depending on individuals' foreign language proficiencies, trigger both lower and higher comprehension. Variation in comprehension is, as a second step and independent of its cause, negatively associated with individuals' tendencies to cooperate. Our experimental results support our theorizing. This study provides new micro-foundations for strategic decision-making and discusses unreliable cooperation as a potentially destructive managerial group dynamic within foreign language contexts.
\end{abstract}

Keywords Foreign language use · Information comprehension · Judgment and decision-making $\cdot$ Strategic decision-making $\cdot$ International management

\section{Introduction}

Global business operations and an increasingly diverse workforce imply that an ever-increasing number of managers and employees are required to use languages other than their native tongue in the workplace (Frederiksson et al. 2006; Neeley 2012; Tenzer et al. 2017). However, many individuals fear that their comprehension of relevant information-that is, the extent to which relevant information is understood-might suffer when using a foreign language. Such an actual or expected lack

Diemo Urbig

urbig@b-tu.de

Extended author information available on the last page of the article 
of information can hamper inter-organizational and intra-organizational processes (e.g., Harzing and Feely 2008; Harzing et al. 2011; Neeley 2013; Neeley and Dumas 2016; Piekkari et al. 2005; Tenzer et al. 2014). Complementing these effects at the group level, using a foreign language also changes how individuals decide (e.g., Akkermans et al. 2010; Costa et al. 2014b; Keysar et al. 2012; Urbig et al. 2016). An emerging stream of research argues that foreign language use changes individuals' inclinations to engage either in intuitive and heuristic or in reflective and deliberate thinking (e.g., Costa et al. 2014a; Keysar et al. 2012; Urbig et al. 2016). However, these studies do not directly relate foreign language use to reductions in comprehension. Usually, they empirically ensured a complete understanding, thereby leaving aside the fact that a decrease in comprehension has been suggested to constitute the critical problem that triggers changes in thinking when using a foreign language (Alter et al. 2007; Costa et al. 2014b).

To advance international business and global strategy research, we develop and test a conceptual framework that explicitly focuses on comprehension. Drawing on dual-process theories (e.g., Stanovich 2009; Thompson 2009), which are often employed to explain behavior in foreign language contexts (e.g., Keysar et al. 2012; Volk et al. 2014), we propose that comprehension fulfills a mediating role. In so doing, we respond to calls to hypothesize more explicitly regarding the information extracted from a stimulus and to frame related triggers to deliberate thinking in terms of basic comprehension processes (Thompson 2009). We conceptualize comprehension as the extent to which the receiver of a message believes to have understood the information contained (McGrath 2001). That is, comprehension represents a state after having invested effort to understand a specific piece of information. While language proficiency and fluency that may affect comprehension have received substantial attention in research on foreign language and decision-making (e.g., Alter and Oppenheimer 2009; Hayakawa et al. 2016; Volk et al. 2014), comprehension as a distinct construct is less well studied. Concerning processes of understanding and re-contextualizing within communication across international, cultural, and linguistic borders (Brannen 2004), our focus is on the informational and uncertainty-reducing aspect of communication (Sullivan 1988).

For the empirical part of our study, we adopt an experimental design, specifically focusing on strategic behavior in the context of Bertrand price competition, which is a particular and, in business practice, a relevant instance of a Prisoner's dilemma. The Prisoner's dilemma mirrors strategic decision-making in many situations that feature a tension between individual and collective rationality (e.g., Boone et al. 1999; Jones and George 1998; Parkhe 1993; Tsai 2002; Zeng and Chen 2003) and it has been successfully employed in research on intercultural contexts (e.g., Akkermans et al. 2010; Cox et al. 1991; Tenzer et al. 2017). In our experiment, participants could opt for cooperative or competitive pricing in duopolistic price competitions. The experiment was administered either in the participants' mother tongue (Dutch) or in a foreign language. We took English as the foreign language, as this is a dominant language in international business, trade, and politics (e.g., Crystal 2003; Feely and Harzing 2003; Ku and Zussman 2010; Neeley 2012).

Our study contributes to research on how foreign-language use affects individual decision-making in two ways. First, our theoretical discussion of the role of 
comprehension identifies previously hidden conceptual ambiguities with respect to different and possibly opposing mechanisms that link foreign language use with individuals' decision-making in a global context. To better understand the effect of foreign language use on decision-making, we follow previous research on decision-making (e.g., Ellsberg 1961; Kahneman and Tversky 1979) and on decision-making in foreign language research (e.g., Hadjichristidis et al. 2017), explicitly distinguishing between information processing, which leads to more or less comprehension, and decision-making, which builds on the comprehended information. We emphasize that deliberate and reflective information processing, which can be activated by using a foreign language (Costa et al. 2014a), is more than intuitive decision-making associated with a high cognitive load (cf., Evans and Stanovich 2013). If information processing and decisionmaking compete for mental resources, then cognitive load due to more deliberate information processing might lead to less cognitive capacity available for decision-making, and hence to more intuitive rather than deliberate decision-making. Our conceptual focus on comprehension, which separates information processing from decision-making, and our consideration of the specific timing of these processes (cf., Hadjichristidis et al. 2017) in tandem enables us to demonstrate how foreign language use can be theorized to induce both less (e.g., Costa et al. 2014a; Keysar et al. 2012) and more (e.g., Takano and Noda 1993; Volk et al. 2014) heuristic thinking.

Second, empirically, we document conditional causal effects of foreign language use on both comprehension and cooperation with the direction of the effect depending on individuals' foreign language proficiency. Our finding that using a foreign language may increase comprehension when individuals are highly proficient in the foreign language provides evidence for the previously suggested deliberation-triggering effect of foreign language use (Costa et al. 2014a; Keysar et al. 2012; Urbig et al. 2016). While these previous studies mostly provide only indirect evidence based on interpreting related decision-making, this study focuses on the direct outcome of a more deliberate information processing, that is, an increase in comprehension. Furthermore, by demonstrating comprehension as an essential mediator of the relationship between foreign language use and individual behavior, we also introduce a new mechanism into the discussion of how language use affects information processing and decision-making in a variety of managerial settings, both in international business and in multilinguistic organizations. Considering information comprehension as a construct allows research on foreign language use to draw on and integrate previously neglected streams of research on decision-making, including, as we demonstrate, Ellsberg's (1961) work on ambiguity, which relates to comprehension through its reference to reliability and 'unanimity' of information that eventually affects their decision-making. Thereby, our study helps scholars and practitioners alike to deepen their understanding of the mechanisms and related effects of communication across linguistic boundaries in today's global managerial world.

\section{Theory and Hypotheses}

The use of foreign language in international business contexts is gaining increasing attention-see, among others, the review by Tenzer et al. (2017) and the special issue of the Journal of International Business Studies focusing on this topic 


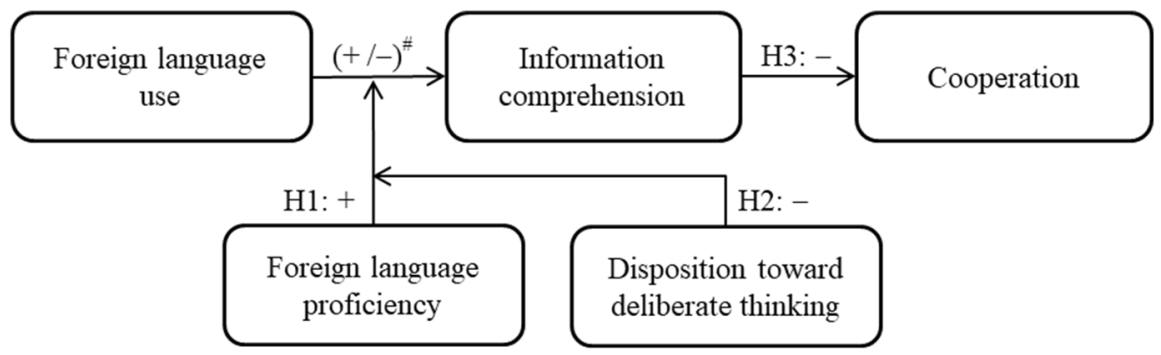

Fig. 1 Conceptual overview. Notes: "Expected to be negative on average, but depending on the moderator, foreign language proficiency, can also be positive for people with very high foreign language proficiency

(Brannen et al. 2014). Across various disciplines, researchers show that foreign language use affects individuals' decision-making (Costa et al. 2014a), including individuals' loss aversion (Keysar et al. 2012), as well as their ethical and social behaviors (Costa et al. 2014b; Geipel et al. 2016; Urbig et al. 2016). There are multiple mechanisms considered relevant in explaining such effects. Foreign language use may change the extent to which individuals base decisions either on intuitive and heuristic or on reflective and deliberate reasoning (Costa et al. 2014a; Hayakawa et al. 2016; Volk et al. 2014). In addition, reduced emotions and increased psychological distance are also discussed as possible mechanisms underlying observed foreign language effects. In contrast to studies comparing the explanatory power of different theoretical accounts (e.g., Hayakawa et al. 2017; Vives et al. 2018), we focus on the intuition-deliberation perspective (cf., Costa et al. 2014a; Hayakawa et al. 2016; Volk et al. 2014) and study the role of information comprehension as a crucial mediator between information processing and decision-making.

An individual's information comprehension refers to subjective evaluations of situations or stimuli (see McGrath 2001, for a related team-level definition). Comprehension is a crucial aspect of an individual's subjective belief about how reliable the information is which enters into a decision. Comprehension of a foreign language stimulus - which, for ease of exposition, we refer to as comprehension-results from an interplay of the individual-specific and stimulus-independent language proficiency with a specific and possibly difficult to understand foreign language stimulus. Depending on the ease with which an individual can establish a high level of comprehension, a foreign language stimulus might be described as being associated with a lower or higher fluency (Alter and Oppenheimer 2009). If an individual experiences high fluency across a large and diverse set of foreign language stimuli, we would argue this individual displays high foreign language proficiency. Whether or not an experience of low fluency results in low comprehension depends on how an individual deals with it. Hence, comprehension is distinct from both language proficiency and fluency. In our conceptual framework (see Fig. 1), comprehension is affected by information processing (Thompson 2009) and feeds into decision-making processes (Ellsberg 1961). Comprehension, thereby, acts as a mediator that helps to conceptually separate information processing, which forms perceptions of behavioral options 
and related outcomes, from evaluation and decision processes (Ellsberg 1961; Hadjichristidis et al. 2017; Kahneman and Tversky 1979; Tversky and Kahneman 1992).

According to dual-process theories (Alter et al. 2007; Evans and Stanovich 2013; Kahneman 2011), individuals possess two distinct types of reasoning. First, Type 1 processes are intuitive, fast, heuristic, automatically cued, and require little cognitive effort. The defining feature of Type 1 processes is that they do not require (substantial) working memory capacity (Evans and Stanovich 2013) and, thus, can serve as default intuition. This intuitive reasoning is effective when individuals perform behaviors that are innate or have been internalized through extensive training experience, such as tying one's shoelaces or communicating in one's mother tongue. However, when confronted with novelty or difficulty, such as programming a new software tool or communicating in a foreign language, individuals might sense that their intuitive and heuristic response is insufficient or inappropriate. This difficulty may prompt the intervention of reflective reasoning on the default intuition and a re-analysis of the problem. Second, the intervening Type 2 processes are reflective, deliberate, systematic, often slower, and requiring more effort cognitively. The defining feature of Type 2 processes is that they require substantial working memory capacity (Evans and Stanovich 2013). Individuals who anticipate difficulties can respond by adjusting cognitive effort and by utilizing more working memory in order to improve their reasoning through having reflective Type 2 processes overrule and correct outcomes generated by the intuitive and heuristic Type 1 processes. Hence, outcomes of intuitive Type 1 thinking also serve as input, and possible anchor, for reflective Type 2 reasoning processes (Evans and Stanovich 2013). If the reflective Type 2 reasoning is active but does not provide a compelling response due to, for example, cognitive overload or if lacking substantial information, the individual will—despite employing reflective Type 2 reasoning - rely on the response generated by the intuitive Type 1 thinking.

Dual-process research is beginning to acknowledge the critical role that comprehension-or, more precisely, the perceived lack of comprehension-might play in activating deliberate information processing (Alter et al. 2007; Evans and Stanovich 2013; Thompson 2009). They also emphasize improved comprehension as an outcome of such deliberate information processing. Consequently, they urge that " $[\mathrm{t}] \mathrm{he}$ next level of theorizing needs to be explicit about the information that is extracted from a stimulus... and frame the issue in terms of basic comprehension processes" (Thompson 2009, p. 185). This request coincides with calls from management and international business scholars to pay closer attention to communication theories and related comprehension processes in global business environments (e.g., Brannen 2004; Brannen et al. 2014; Tenzer et al. 2017). Previous studies applying dual-process theories to study the effects of foreign language use on individuals' decisionmaking, however, do not explicitly address comprehension. Conceptual frameworks tend to abstract from comprehension processes and instead focus on working memory load (e.g., Volk et al. 2014). So far, experimental studies on the effects of using a foreign language mostly seek to minimize the influence of variation in comprehension, either by focusing on participants with almost perfect comprehension (e.g., Costa et al. 2014a, b; Keysar et al. 2012; Urbig et al. 2016) or by considering secondary unrelated tasks that do not depend on the comprehension of foreign language stimuli (e.g., Takano and Noda 1993, who study math calculations while 
being interrupted by unrelated language-related questions). Despite its relevance to dual-process theories, the role of comprehension is understudied for the effects of using a foreign language on behavior.

\subsection{Foreign Language Use and Reduced Comprehension}

The primary function of using a foreign language in international business settings is to allow individuals to communicate with each other even when their mother tongues differ (Chidlow et al. 2014; Sullivan 1988). When speaking a nonnative language, however, individuals tend to experience difficulties related to both encoding and decoding information. They may suffer from reduced communication abilities and a loss of rhetorical skills due to limited vocabulary and complex grammatical structures (e.g., Birdsong 2006; Harzing and Feely 2008) or differences in contextualization conventions across languages (Brannen 2004; Gumperz and Gumperz 1996). Consequently, using a foreign language may reduce the comprehension of available information. The difference in comprehension depends, among others, on an individual's proficiency in the foreign language (e.g., Birdsong 2006)—i.e., the higher a person's proficiency, the smaller the potential loss in terms of comprehension.

Humans, however, are highly adaptive and adjust their information processing and decision-making in order to accommodate cognitive demands posed by their environments (Alter et al. 2007; Evans and Stanovich 2013; Kahneman 2011). The command of two distinct types of reasoning - the intuitive and heuristic Type 1 and the reflective and deliberate Type 2 reasoning - allows for such adjustments. Processing difficulties and (linguistic) disfluency evoke more deliberate and systematic thinking processes (Alter and Oppenheimer 2009). By devoting more mental capacities to processing the foreign language, individuals can extract more information, thereby mitigating potential disadvantages resulting from initial processing difficulties. ${ }^{1}$ That is, when confronted with communication in a foreign language, which carries a higher risk of misunderstanding compared to communication in the native language, individuals tend to process the information in a more effortful manner, switching to a deliberative thinking mode. Thus, foreign language use has a deliberation-triggering effect (Costa et al. 2014a; Keysar et al. 2012; Urbig et al. 2016). The language-induced cognitive load may, therefore, not necessarily act as an obstacle to information processing in foreign language contexts, but constitutes a part of the solution to overcome the linguistic disfluency.

Devoting more cognitive effort to foreign rather than native language stimuli is shown to cause bilingual individuals to perform objectively better in linguistic tasks (e.g., higher accuracy and speed of recognition when learning lists of words) in the less compared to the more fluent language (Francis and Gutiérrez 2012). That is,

\footnotetext{
1 Importantly, it is the subjective experience of potential or actual deficiency in comprehension that eventually affects individuals' behavior (e.g., Neeley 2013) and their cognitive responses to related stimuli (e.g., Alter and Oppenheimer 2009). Individuals exaggerating their comprehension are likely to engage in similar cognitive processes as those who correctly perceive high levels of comprehension; however, their decisions are based on possibly different and misperceived information.
} 
deliberately processing a foreign language cue may, surprisingly, lead to even better comprehension than intuitively processing a native language cue. Independent of objective levels of comprehension, the mental investment in more deliberation associated with processing a foreign language cue could trigger higher subjective levels of confidence in the extracted information (Alter and Oppenheimer 2009). This comprehension-increasing effect, however, depends on individuals' foreign language proficiencies. To be successful in overcoming a language-induced lack of comprehension or even increase comprehension beyond its level in native language contexts, individuals need a rather high proficiency in the foreign language.

For individuals with low proficiency, though, using a foreign language is likely to reduce comprehension. Foreign language use may initially trigger a switch toward deliberate Type 2 information processing, as suggested above. However, a pronounced lack of language proficiency is likely to prevent the reflective Type 2 reasoning from achieving levels of comprehension that match the corresponding comprehension of a native language stimulus. That is, even the highest levels of mental effort cannot counter the drawback of not having understood the foreign language stimulus. In such cases, individuals may fall back to representations and outcomes provided by the intuitive Type 1 processes (Thompson 2009). Consequently, low proficiency individuals are stuck with lower comprehension in the foreign language context.

Hypothesis 1: When using a foreign language rather than a native language, individuals with low language proficiency experience a decrease in comprehension, whereas highly proficient individuals experience a smaller decrease, if not an increase, in comprehension.

\subsection{Individuals' Dispositions Toward Deliberate Thinking}

At the core of our conceptual framework is a shift between employing the intuitive and heuristic Type 1 and the reflective and deliberate Type 2 thinking processes. Dual-process theories suggest that individuals' predispositions influence their inherent likelihood to employ either one of these two types of processing in their thinking and decision-making (Stanovich 2009; Thompson 2009). Some individuals are more prone than others to override their intuition in favor of deliberate reasoning. As individuals with a stronger disposition toward reflective reasoning are generally more likely to rely on reflective Type 2 processes, additional deliberation-triggering effects, such as those effectuated by using a foreign language, are less likely to affect them.

In contrast, such a deliberation-triggering effect is likely to be stronger for individuals with a disposition toward intuitive processing. These individuals' deliberate thinking tends to be inactive, requiring additional stimulation to be activated. In support of such an effect, Urbig et al. (2016) empirically show that an individual's disposition toward deliberate thinking attenuates the effect of foreign language use on behavior. Thus, we expect an individual's predisposition to mitigate the previously hypothesized deliberation-triggering effect of foreign language use. 
Hypothesis 2: The proficiency-moderated relationship between foreign language use and comprehension is weaker for individuals who are predisposed toward deliberate thinking compared to those who are predisposed toward intuitive thinking.

\subsection{Comprehension and Cooperation Behavior}

But having exploited knowledge, guess, rumor, assumption, advice, to arrive at a final judgment that one event is more likely than another or that they are equally likely, one can still stand back from this process and ask: "How much, in the end, is all this worth? How much do I really know about the problem? How firm a basis for choice, for appropriate decision and action, do I have?" (Ellsberg 1961, pp. 659-660).

Referring to Ellsberg (1961), we assume that, at some point, individuals have completed all their efforts to process the task information; that is, they have achieved some level of comprehension regarding the issue at hand. What is left at this stage is to take the actual decision, based on whatever subjective knowledge or comprehension the person has arrived at (see also Kahneman and Tversky 1979). In the current study, we turn our attention to the relationship between comprehension and cooperation - a relationship that is, for example, of particular relevance for strategic decision-making regarding cooperation with (potential) competitors in a globalized business context. In the following, we link less comprehension to cooperation decisions through differences in individuals' tendencies to engage in less deliberate and more intuitive decision-making.

In order to link comprehension to the type of thinking, we build on established research on ambiguity, specifically the work by Ellsberg (1961) as well as Fox and Tversky (1995). Individuals who perceive a lack of comprehension tend to experience ambiguity (cf., Ellsberg 1961; Fox and Tversky 1995). Experiencing ambiguity that is not resolved through deliberation, in turn, tends to induce a more heuristic, intuitive, and experimental, rather than a deliberate and analytic approach (Mosakowski 1997; Slovic et al. 2007). That is, if individuals believe they are-despite having tried to avoid it-suffering from a lack of comprehension, they are more likely to approach a decision-making task in a heuristic manner. Consequently, comprehension at the time of decision-making is positively associated with more deliberate and reflective decision-making, and a perceived lack of comprehension is associated with more intuitive and heuristic decision-making.

The described general mechanism associating a lack of comprehension with more intuitive and less deliberate decision-making can become even more pronounced when individuals' engagements in both processing information about the decisionmaking consequences in the foreign language and decision-making based on this information are cognitively demanding. If both these tasks are in close temporal proximity, they may compete for limited cognitive capacity (Just and Carpenter 1992; Takano and Noda 1993). Assuming that mental capacities, such as working memory (e.g., Volk et al. 2014), are constrained, engaging in multiple tasks may 
exhaust the overall available mental capacity, resulting in cognitive overload. Putting more capacity into one task, such as foreign language processing, results in a depletion of mental capacities available for the remaining tasks-for example, the focal decision-making (Evans and Stanovich 2013; Thompson 2009; Volk et al. 2014). Cognitive overload may render the task with decreasing mental capacities, which is decision-making in this case, more intuitive (e.g., Takano and Noda 1993). Individuals who cannot ensure high levels of comprehension are more likely than others to experience such an overload-induced shift to intuitive decision-making. Individuals who successfully ensure high levels of comprehension, in contrast, are less likely to experience such an overload. They may not have exhausted the available mental capacity at all, even when almost simultaneously engaging in both foreign language processing and decision-making. In this situation, the mobilization of additional cognitive capacity to cope with foreign language processing may also draw more cognitive capacity (out of the free mental capacity) into the related decision-making (Alter and Oppenheimer 2009; Keysar et al. 2012; Urbig et al. 2016). Due to this spillover of the alerting effect of using a foreign language, individuals with better comprehension could become even more deliberate in their decision-making in a foreign language context, which, in sum, further strengthens the positive association of comprehension with deliberate decision-making.

We next consider which effect a more intuitive rather than deliberate mode of thinking might have on the individual's decisions regarding whether or not to cooperate. This question is at the heart of a large stream of research within cognitive science, which seeks to understand the puzzling ubiquity of humans' prosocial and cooperative tendencies (e.g., Rand 2016; Zaki and Mitchell 2013). In investigating this question, scholars argue that-when relying on intuitive decision-making - the majority of people tend to resort to social norms as a powerful heuristic (Rand et al. 2014; Weber and Murnighan 2008) and that social norms tend to favor cooperation over non-cooperation: While the individual's rational decisions in a social dilemma is to opt against cooperation, many humans empirically refrain from fully exploiting others (e.g., Bolton and Ockenfels 2000; Fehr and Schmidt 1999). Instead, they decide to cooperate, at least to some extent. Thus, it is likely that cooperative behavior constitutes a strong social norm.

Consistent with this substantial body of work that associates intuitive decisionmaking with a relatively higher prevalence of cooperation (e.g., Cornelissen et al. 2011; Duffy and Smith 2014; Rand 2016, 2019; Rand et al. 2012), we expect that individuals' intuitive and heuristic response to a perceived lack of comprehension is characterized by a higher tendency to cooperate. Note that, while the strength and possibly even the specific content of social norms may depend on culture (e.g., Capraro and Cococcioni 2015), we believe this specific argument to hold for the Western-European context of this study. ${ }^{2}$ Putting together the discussion of how comprehension relates to intuitive versus deliberate decision-making, and how the level of intuitiveness and deliberativeness of decision-making relates to cooperative behavior,

\footnotetext{
${ }^{2}$ This is especially true for the Dutch context, which is thought to reflect a society that is more cooperative (in terms of social norms) and less masculine than most other Western-European countries (cf., Akkermans et al. 2010).
} 
we, therefore, expect that less comprehension is associated with more cooperation. The notion of a greater inclination to cooperate in the presence of comprehension deficiencies is also consistent with Andreoni's (1995) culture-independent suggestion that confusion, in general, might lead to cooperative behavior. Specifically, factors that increase confusion, like reduced comprehension, may lead to cooperation in settings where individuals would rationally not cooperate. In sum, this leads to our last hypothesis linking less comprehension with more cooperative behavior.

Hypothesis 3: Less comprehension is associated with more cooperation.

\section{Method}

To test our hypotheses, we employ an experimental approach to identify empirically the fundamental causal effect of foreign language use on comprehension and cooperation (Zellmer-Bruhn et al. 2016; for a similar approach, see Cox et al. 1991). We experimentally randomize the language of presentation (foreign versus native). To capture individuals' strategic cooperation behavior, participants are exposed to a prisoner's dilemma (e.g., Boone et al. 1999; Chen and Li 2005; Cox et al. 1991; Parkhe 1993; Zhang and Rajagopalan 2002), which is characterized by an inherent tension between gains from cooperation (maximizing collective payoffs) and incentives to free-ride on partners who cooperate (maximizing individual payoffs). To increase external validity, we frame the prisoner's dilemma as duopolistic price competition between two firms (Akkermans et al. 2010). To increase the relevance of the decision for participants, we incentivized the experiment through a within- and between-subject random incentive system. A randomly selected subgroup of participants was paid for a randomly selected part of the experiment with real payments between 10 Euro and 490 Euro, depending on participants' decisions in the experiment (the final firm value divided by 10,000).

\subsection{Sample}

Following previous studies using students to investigate cross-national differences in cooperation (e.g., Chen and Li 2005) and equity effects on decision-making in strategic management (Elfenbein et al. 2017), the sample consists of students at a major university in the Netherlands. Participants were enrolled in a business program and followed a compulsory introductory course on organization studies. Dutch individuals generally exhibit relatively high levels of English language proficiency, which allowed us to administer the experiment meaningfully in both languages. At the beginning of the academic year, the students were assigned randomly to tutorial groups consisting of about 30 students each. All students were asked to fill in an online survey (in Dutch) to collect information on socio-demographics, personality traits, as well as language- and culture-related features. Two weeks later, they participated in an experiment that took place during their regular tutorial sessions. Participation was voluntary. Only students who completed both the online questionnaire and the experiment were eligible for the money prizes. To ensure anonymity, we matched survey and experimental data using a unique identifier created by 
the participants from several information items, such as 'first two letters of mother's first name.' To ensure that the Dutch language treatment is indeed a native-language treatment, we excluded nine individuals, who indicated that Dutch is not their native language. The final sample consists of 238 individuals.

Using a student sample is consistent with standard practices employed in studies published in top tier strategy and international business outlets (e.g., Akkermans et al. 2010; Chen and Li 2005; Cox et al. 1991; Elfenbein et al. 2017). Although student samples are easier accessible, they also offer some methodological advantages. Student samples are valid and useful when the nature of the research question relates to basic characteristics and fundamental processes of human nature (see, for example, the editorials by Bello et al. 2009, van Witteloostuijn 2015, and by Zellmer-Bruhn et al. 2016), which is the case in our study that focuses on the impact of foreign language use on fundamental decisions as to whether or not to cooperate. Furthermore, student samples are advantageous when other samples are impossible to collect or relatively more prone to endogeneity concerns (e.g., Bönte et al. 2016; Williams et al. 2019). The homogeneity of student samples, primarily when recruited in the same education program, tends to facilitate homogeneity in beliefs and (social) attitudes when randomly dividing the sample into treatment and control groups, thereby substantially reducing threats from unobserved heterogeneity. This homogeneity also ensures a higher statistical power by holding constant other factors that may additionally increase the heterogeneity in the dependent variable. Problematic, in the case of non-student samples, are employees' partly endogenous experiences resulting from their previous choices, behaviors, and experiences in foreign language contexts. The specific characteristics of the student sample, that is, young age and somewhat similar experiences, allow us to identify the causal effects of using a foreign language more reliably. The salient self-selection of students in our sample-i.e., their choice to opt for a business study program-increases the probability that the results of this study may generalize to real-world settings of organizational relevance, as these students are prospective future employees and managers.

\subsection{Experimental Design}

Inspired by Akkermans et al. (2010), we exposed participants to the following strategic dilemma: Two firms, firms A and B, operate in the same market. The firms are identical, offering the same homogenous product while being equally efficient. Resulting from deep pockets (i.e., 2,500,000 Euro earnings from last year), bankruptcy is not an issue. Both firms can choose between two pricing strategies: setting a low price $(\mathrm{L})$ or a high price $(\mathrm{H})$. Consumers select their preferred product based on price only. The firms' profits depend on the pair of chosen pricing strategies. Participants decided autonomously on the pricing strategy of their respective company and fixed prices for four quarters of a year. This set-up allowed participants to cooperate with the competitor by setting high prices either entirely or only to some extent (for example, in only one of the four quarters), if they wanted to-a design feature that increases variability in responses and, hence, the power to identify effects. Indeed, $38 \%$ of our participants used this opportunity to exhibit nuanced behavioral patterns. 


\begin{tabular}{lll}
\hline & \multicolumn{2}{l}{ Firm B } \\
\cline { 2 - 3 } & Low price & High price \\
\hline Firm A & & \\
Low price & A: $-200,000$ & A: $+600,000$ \\
& B: $-200,000$ & B: $-600,000$ \\
High price & A: $-600,000$ & A: $+200,000$ \\
& B: $+600,000$ & B: $+200,000$ \\
\hline
\end{tabular}

Cells indicate profits in Euro for firm A and firm B

For each quarter of a year, mutual cooperation and mutual non-cooperation yielded payoffs of 200,000 and $-200,000$ Euro, respectively, for each player. If one player cooperated while the other player chose not to cooperate, the first player made a loss of $-600,000$ Euro, whereas the second made a profit of 600,000 Euro (see Table 1).

The experimental sessions took place either in Dutch (the participants' native language) or in English (their foreign language). In Dutch sessions, experimenters were native Dutch speakers, while during the English sessions, experimenters were foreigners unable to speak Dutch. We randomized the language treatments across tutorial sessions, with all written documents and verbal instructions presented in the treatment language. We truthfully motivated the use of English by the inclusion of foreign experimenters in the research group who could not communicate in Dutch. Given our research question, which rests on variation in comprehension, and to avoid related confounding effects, we did not implement elaborate mechanisms ensuring perfect understanding of instructions (e.g., Oganian et al. 2016; Urbig et al. 2016), did not exclude participants who had not fully understood the rules of the experiment (e.g., Geipel et al. 2016), and did not measure participants' objective understanding by letting them translate the instructions after the experiment to exclude those displaying insufficient understanding (e.g., Costa et al. 2014a; Keysar et al. 2012). ${ }^{3}$

\subsection{Model Variables}

Cooperation is operationalized as the relative frequency of cooperative choices over the four quarters of a year. The values range from zero for no cooperative choice, over 0.25 (one cooperative choice), 0.5 (two), 0.75 (three), to 1.0 for cooperative choices in all four quarters.

Comprehension, as a subjective and context-specific construct, is operationalized by averaging two responses to the following question, with one response obtained during and one obtained at the end of the experimental treatment: "Given that the experiment was pretty complex, what percentage (0-100) of the questions/text/game

\footnotetext{
3 Note that correctly translating experimental instructions after the experiment does not necessarily imply that participants have correctly understood the instructions during the experiment. The explicit call for translation might, consistent with dual-process theories, trigger additional mental effort, whichcompared to reading instructions during the experiment-may lead to a higher level of comprehension. Furthermore, this objective measure of comprehension does not shed light on subjective perceptions, which are likely to be more relevant for behavioral choices, such as whether or not to cooperate.
} 
did you fully understand?" The item content resembles McGrath's (2001) team-level measure of comprehension. Asking the question twice ensures that the responses capture the comprehension during the whole experiment and not just the specific part of the experiment preceding one of these measures. By averaging the two measures, we also reduce random measurement errors associated with every single measurement, thereby increasing the reliability of the measure of comprehension. Using only the response from the question directly after their cooperation decisions, however, does not change our conclusions (estimations available upon request). The average of the inverse of these two responses $(\alpha=0.86)$ was divided by 100 , such that one reflects perfect comprehension, and zero reflects a complete lack thereof.

The language treatment is coded as a dummy variable English, with ones and zeros indicating English and Dutch treatments, respectively.

We measure foreign language proficiency through participants' responses to "How do you describe your own ability to understand English?" on a seven-point scale ranging from 'very poor' (1) to 'excellent' (7). While such self-reported data may not perfectly capture an individual's actual competence, it does provide an appropriate measure in our experimental setting because subjectively perceived proficiency is what matters for individuals' decision-making processes (e.g., Neeley 2013).

We approximate participants' disposition toward deliberate thinking by their conscientiousness (Urbig et al. 2016), an established personality trait with an established measurement instrument. According to both the Big-Five and the HEXACO personality frameworks, conscientious individuals organize their time and their physical surroundings rather than being sloppy and haphazard, they work in a disciplined way toward their goals, strive for accuracy and perfection in their tasks rather than tolerate errors in their work and neglect details, and they carefully deliberate when making decisions rather than being satisfied with work that contains some errors, and with making decisions made impulsively or with little reflection (Lee and Ashton 2004; McCrae and John 1992). While the latter aspect specifically focuses on deliberative versus impulsive approaches, the other aspects are also reflective of individuals who are willing to pay attention and invest (cognitive) effort also in situations where other people would not do this. Hence, we argue that while conscientiousness is not a perfect measure for the disposition toward deliberate thinking, it constitutes a suitable proxy. ${ }^{4}$ We employed the conscientiousness subscale of the HEXACO personality inventory (Lee and Ashton

\footnotetext{
4 Self-reported measures of individuals' dispositions toward deliberate thinking tend to focus on the defining feature of cognitive capacities devoted to reflecting and decision-making. Peysakhovich and Rand (2016) alternatively use the cognitive reflection test (Frederick 2005) to measure individuals' disposition toward deliberate thinking. While suffering less from biases associated with self-reported measures, these behavioral measures indirectly derive the disposition from particular outcomes of thinking processes. Given that even reflective and deliberate thinking may lead to decisions outcomes suggested by intuitive processes (Evans and Stanovich 2013; Thompson 2009) and, additionally, are highly susceptible to biases associated with the high specificity of the test situation, following Urbig et al. (2016), we decided to rely on the well-established measurement of conscientiousness as a proxy for individuals' disposition to reflective and deliberate thinking. It is important to note that an individual's "disposition for deliberate thinking" is not a situational construct, but a dispositional construct that is relatively stable across variations in time and situation, such that relating the measurement to personality traits is appropriate.
} 
2004), consisting of ten Likert-type items with responses from 1 ('strongly disagree') to 5 ('strongly agree'). The scale achieves sufficient internal reliability $(\alpha=0.83)$.

\subsection{Control Variables}

While randomization of language treatments, on average, provides a safeguard against systematic biases in observed manipulated variables' effects due to individual-level heterogeneity, including related control variables increases the power of the analyses by controlling for otherwise random variation, helping to filter out confounding effects on variables not experimentally manipulated. As demographic control variables, we include age (in years) and gender (1 indicates female; 0 indicates male). Additionally, conscientiousness can, and in our data does, correlate with the five remaining personality dimensions of the HEXACO personality inventory. To reduce the likelihood of the effect of conscientiousness being confounded by these other personality traits, we included the remaining five dimensions as control variables (each based on ten Likert-type items with responses ranging from 1 to 5, as for conscientiousness): honesty-humility $(\alpha=0.78)$, emotionality $(\alpha=0.82)$, extraversion $(\alpha=0.78)$, agreeableness $(\alpha=0.72)$, and openness to experience $(\alpha=0.74)$.

As existing research shows that the processing of a foreign language is affected by whether or not the acquisition of the language started during very early childhood (e.g., Hernandez and Li 2007), we control for Late foreign language acquisition $(0=$ until 5 years; $1=$ above 5 years $)$.

Chen and $\mathrm{Li}$ (2005) note the importance of adding individual-level variables of cultural value orientation when investigating cooperation in multicultural contexts. Therefore, we additionally control for individuals' English cultural-linguistic identity, defined as the extent to which participants positively identify with the culture they associate with the foreign (here: English) language (see Bordia and Bordia 2015, for a general discussion of a person's linguistic identity). To assess participants' English cultural-linguistic identity, we adapted a graphical measurement of identification with organizations (Shamir and Kark 2004) to the linguistic context. Participants selected a graph that best represented their relationship to the culture they associate with the English language. Seven graphs were available, which differed in the degree to which two circles overlapped (one for the self and one for the associated culture). We assigned the value of 1 to the graph with no overlap (indicating no identification with the foreign culture) and 7 with full overlap (indicating the highest possible level of identification with the foreign culture); other graphs were assigned in-between values corresponding to the degree of the overlap of the two circles.

Table 2 reports the summary statistics and bivariate correlations. Correlations below 0.5 and variance inflation factors lower than 3.3 indicate that multicollinearity is not a problem (Cohen et al. 2003). However, despite the randomization of participants across language treatments, accidentally, there is a small and statistically significant correlation between language treatments and gender. Thus, statistically controlling for related gender differences is necessary and implemented in our regression analyses. 


\section{Results}

For a descriptive overview of the response patterns observed in our data, Fig. 2 plots the average responses and related standard errors for the Dutch and English language treatments, as well as for subgroups varying in foreign language proficiency and comprehension. Figure 2a illustrates that only individuals with low proficiency experienced a decrease in comprehension when exposed to English, whereas highly proficient participants even experienced a minor increase in comprehension. Furthermore, Fig. 2b shows that a high level of comprehension is associated with a lower tendency to cooperate. Already these subgroup comparisons are consistent with our theory, especially with hypothesis 1 and hypothesis 3 .

\subsection{Hypotheses Tests}

We employ ordinary least squared (OLS) regression analyses to test our hypotheses statistically (see Table 3). We standardize interval-scaled variables to simplify the interpretation when including interaction terms (Cohen et al. 2003) and improve the comparability of effects (Dalal and Zickar 2012). To test the general impact of foreign language use on comprehension, we first estimate a model with control variables and the foreign language treatment effect (Model 1). In English, participants, on average, report lower comprehension, but the effect is not statistically significant. Model 2 includes the moderating effect of foreign language proficiency. In support of hypothesis 1, suggesting that foreign language use decreases comprehension for those with low foreign language proficiency, but might even leverage comprehension for those with high levels of proficiency, the moderation effect is positive and statistically significant. We calculate the conditional effects for individuals with low levels (mean minus one standard deviation) and high levels (mean plus one standard deviation) of foreign language proficiency, respectively. The effect is statistically significant for both conditions: Foreign language use decreases comprehension for those with low levels of proficiency (Model 2: $\beta_{\text {English }}-\beta_{\text {English } \times F L P}=-0.55, \mathrm{SE}=0.18$, $\mathrm{p}=0.002$ ) and increases comprehension for highly proficient individuals (Model 2: $\left.\beta_{\text {English }}+\beta_{\text {English } \times \text { FLP }}=0.35, \mathrm{SE}=0.18, \mathrm{p}=0.049\right)$.

Hypothesis 2 suggests that an individuals' disposition toward deliberate thinking would moderate the effect predicted by hypothesis 1 . To test this moderating effect, we include a corresponding moderation effect related to both the effect of foreign language use on comprehension and its interaction with foreign language proficiency, which results in a three-way interaction effect (Model 3). The three-way interaction between English, foreign language proficiency, and disposition toward deliberate thinking is statistically significant at the level of $\mathrm{p}<0.10$.

For a better interpretation of these results regarding hypothesis 1 and hypothesis 2, Fig. 3 graphically illustrates the language effects for different levels of proficiency and disposition toward deliberate thinking. For illustrative purposes, the dependent variable, information comprehension, is not reported in its standardized form (as in regression tables), but in original unstandardized scores. For each point estimate, 


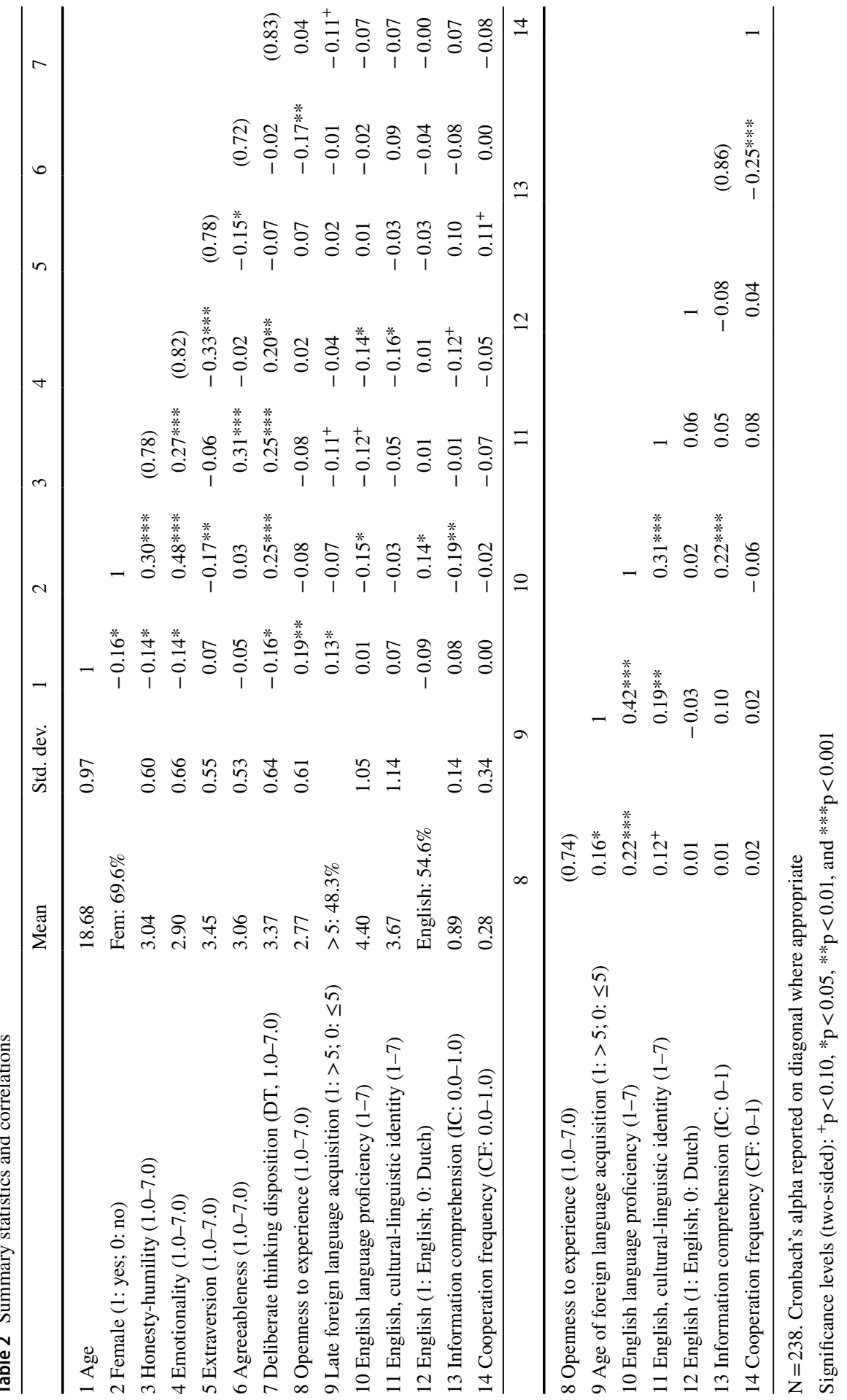



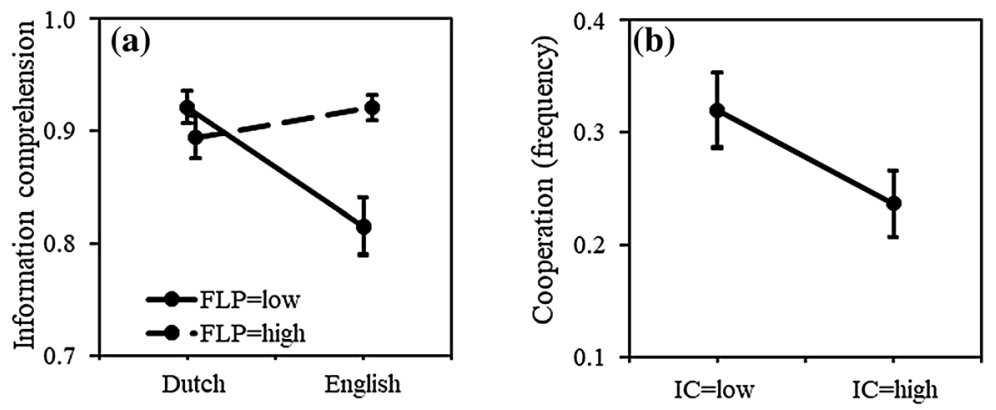

Fig. 2 Treatment and subgroup comparison for information comprehension and cooperation. Notes: Subgroups are based on treatment groups and median splits for low vs. high levels of foreign language proficiency (FLP) and information comprehension (IC). We report average responses per subgroup and related standard errors

we also plot the standard error. Consistent with hypothesis 1, Fig. 3a, b show that comprehension in the English treatment is lower for those with low proficiency, but can be higher for those with high levels of proficiency. Figure $3 a$, b also reveal that low proficiency individuals with a low disposition toward deliberate thinking experience a larger reduction in comprehension when using a foreign language than those with a high disposition toward deliberate thinking. The corresponding slopes of the solid line in Fig. $3 \mathrm{a}$ is $-0.12(\mathrm{SE}=0.04, \mathrm{p}=0.002)$ and of the solid line in Fig. $3 \mathrm{~b}$ is -0.03 ( $\mathrm{SE}=0.03, \mathrm{p}=0.274$ ) for Fig. 3b. Furthermore, highly proficient individuals with a low disposition toward deliberate thinking experience a larger increase in information comprehension than their counterparts with a smaller disposition toward deliberate thinking. The corresponding slopes of the dotted line in Fig. 3a is $0.07(\mathrm{SE}=0.03, \mathrm{p}=0.037)$ and of the solid line in Fig. $3 \mathrm{~b}$ is 0.02 ( $\mathrm{SE}=0.043$, $\mathrm{p}=0.521$ ) for Fig. $3 \mathrm{~b}$. This three-way interaction suggests that-consistent with hypothesis 3-an individual's level of disposition toward deliberate thinking attenuates the effect of foreign language use on comprehension.

Next, we estimate a model for cooperation with control variables, English language dummy, and comprehension (Model 4). In support of hypothesis 3, comprehension decreases the tendency to cooperate in a statistically significant way ( $p<0.001)$; Fig. 3c visualizes this negative effect of comprehension on cooperation.

The combination of hypothesis 1 and hypothesis 2 with hypothesis 3 suggests a moderated mediation effect of foreign language use on cooperation. Following standard procedures for testing moderated mediation effects (Preacher et al. 2007), in Model 5, we additionally include all moderation effects from the first stage-i.e., language effect moderated by foreign language proficiency and by the disposition toward deliberate thinking. None of these variables, including the language effect, is individually statistically significant (see Table 4), nor are they jointly significant $(\mathrm{F}(4,220)=0.44, \mathrm{p}=0.821)$. Thus, there is no direct effect of the language treatment on cooperation. Instead, comprehension mediates most of the effect of foreign language use on cooperation. To statistically test the indirect effects of foreign language on cooperation through information comprehension, we combine Models 3 and 5 


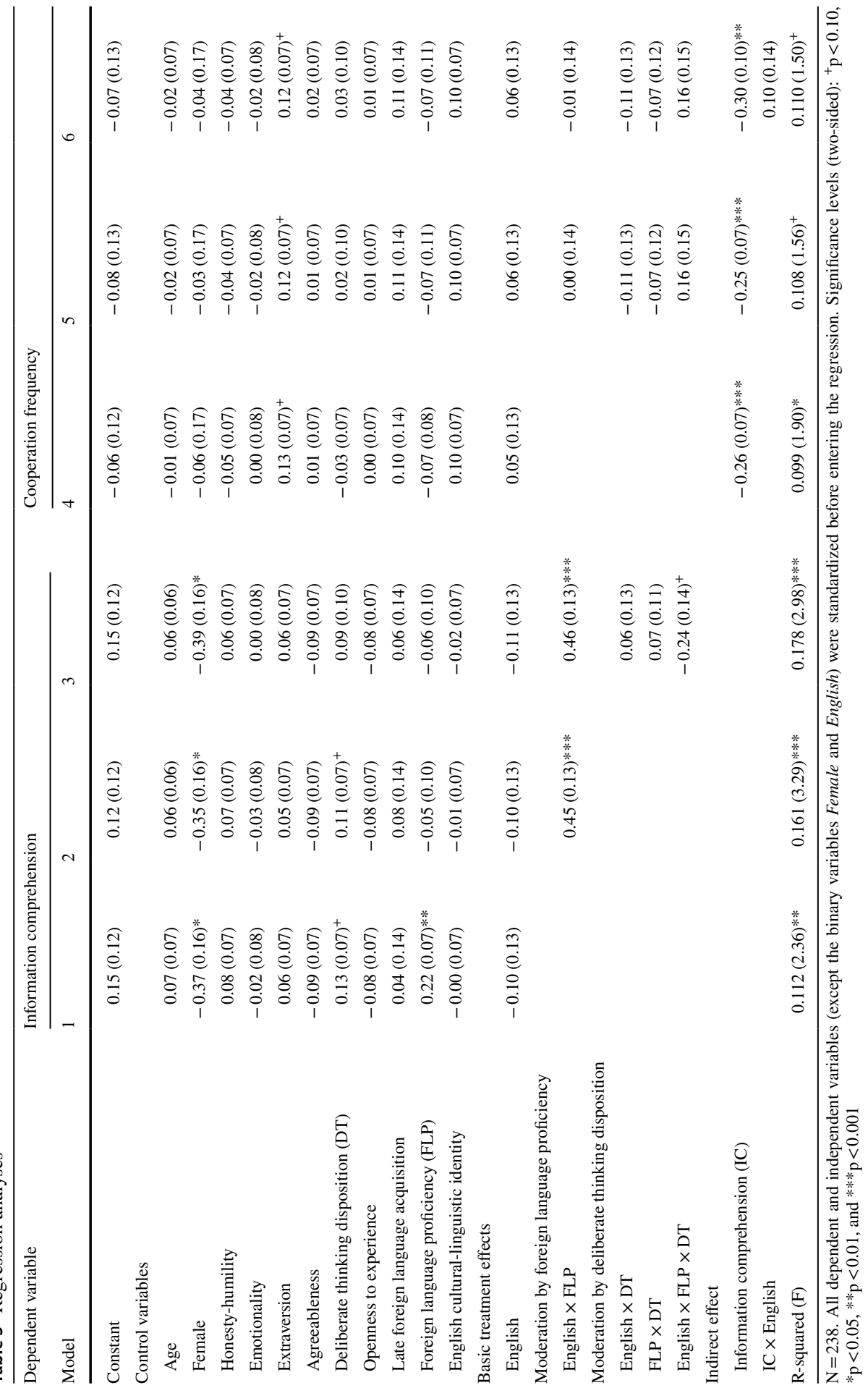



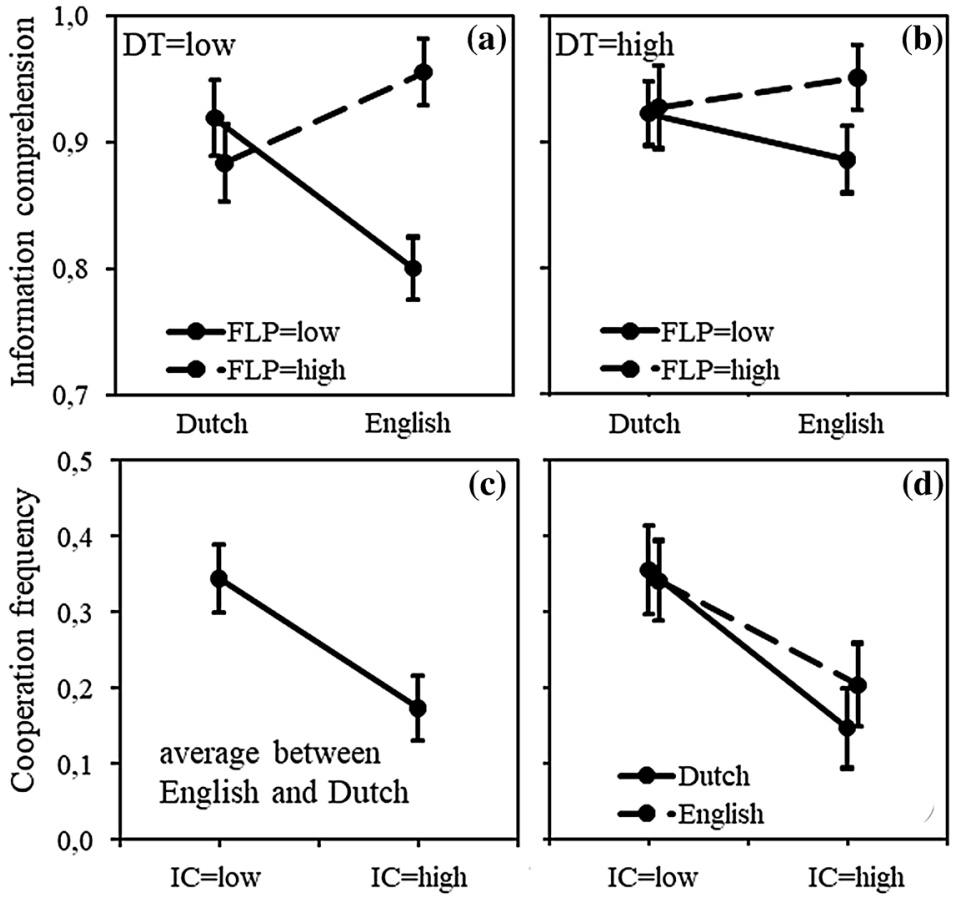

Fig. 3 Estimated interaction effects. Notes: Point estimates for low (mean minus standard deviation) vs. high (mean plus standard deviation) levels of foreign language proficiency (FLP), disposition toward deliberate thinking (DT), and information comprehension (IC). For illustrative purposes, the dependent variable is not reported in its standardized form (as in regression tables), but in unstandardized original scores. For each point estimate, we also plot the standard error

into a structural equation model (available upon request). We calculated indirect effects as the products of the path coefficient conditional upon the levels of foreign language proficiency and disposition toward deliberate thinking. Table 4 reports the effects and related bias-corrected accelerated bootstrapped confidence intervals. When a confidence interval does not include zero, we consider it as support for a significant mediated effect (Preacher et al. 2007). Independent of the level of proficiency and in support of the moderating effect of the disposition toward deliberate thinking, the indirect effects of foreign language use on cooperation through information comprehension are statistically significant for individuals being weakly, but smaller and statistically not significant for those being strongly disposed to deliberate thinking. ${ }^{5}$ In sum, we feel confident that, indeed, the indirect effect of foreign language use on cooperation through comprehension is moderated by foreign language proficiency and individuals' dispositions toward deliberate thinking.

\footnotetext{
5 Due to the randomization of the language treatment, we can causally interpret the conditional effects of foreign language use on both comprehension and cooperation. However, despite following standard practices for quantifying indirect and direct effects of foreign language through comprehension, these statistical mediation analyses are subject to biases and should, hence, not be causally interpreted (Gerber and Green 2012).
} 
Table 4 Conditional indirect effect of English on cooperation through comprehension

\begin{tabular}{lll}
\hline & \multicolumn{2}{l}{ Foreign language proficiency } \\
\cline { 2 - 3 } & Low & High \\
\hline Deliberate thinking disposition & \\
Low & $0.22^{* *}[0.04,0.57]$ & $-0.13^{*}[-0.39,-0.01]$ \\
High & $0.07[-0.04,0.21]$ & $-0.04[-0.27,0.07]$ \\
\hline
\end{tabular}

Calculation of indirect effects of English on cooperation and their bias-corrected accelerated 95\%-confidence intervals (reported in brackets) based on bootstrapping with 4000 repetitions. Significance levels determined based on corresponding confidence intervals not including zero

Significance levels (two-sided): ${ }^{+} \mathrm{p}<0.10,{ }^{*} \mathrm{p}<0.05, * * \mathrm{p}<0.01$, and $* * * \mathrm{p}<0.001$

\subsection{Robustness Checks}

Comprehension might also be influenced by other causes, independent of foreign language use-for example, the complexity of the setting at hand or individuals' levels of involvement. Variation in comprehension that results from such other causes could be associated with different cause-specific effects on cooperation behavior. ${ }^{6}$ To explore such cause-specificity for language as a specific driver of comprehension, Model 6 allows the language treatment to moderate the effect of comprehension on cooperation. Figure $3 \mathrm{~d}$ visualizes the estimated moderation effect; it is tiny and-as reported in Table 3-statistically not significant. Thus, the effect of variation in comprehension on cooperation does not depend on whether it occurs in native or foreign language settings. This finding substantially strengthens our conceptualization of comprehension as a pivotal mediator that separates understanding of the decision context from strategic decision-making.

The Netherlands is a smaller European country. Typically, in such countries, many people speak multiple languages and are used to listening to media, such as TV, in a foreign language. Frequently, in The Netherlands, it is in English. This smallcountry effect implies that many of our participants speak and understand English almost like their native language, or at least believe that they speak and understand it like their native language. However, among the responses to the question regarding their English skills, only six participants out of the sample of 238 reported having very good or excellent skills and, hence, display a level of fluency that would be similar to what one would expect for a native language. Excluding these individuals does not change our conclusions (see Models 7 and 10 in Table 5). The original question regarding the age when participants started learning English allowed separating different age categories, i.e. 'from birth', '0-5 years old', '6-10 years old',

\footnotetext{
${ }^{6}$ With respect to the effects of cognitive load, Volk et al. (2014) introduce such cause-specific effects when suggesting that the effect of foreign language-induced cognitive load (but not other sorts of cognitive load) on heuristic biases is moderated by foreign language proficiency.
} 
'11-16 years old', and at higher ages. Within the sample, nobody indicated having learned it from birth, and only six indicated to having learned it at an age below 6 . Excluding these six individuals does not change our conclusions (see Models 8 and 11). In sum, we can conclude that our results are neither driven by individuals who believe that they can speak English as well as their native Dutch nor by the fact that many of the participants started learning English at a very young age.

Furthermore, by design, the experimental treatments have native language stimuli in the Dutch sessions and foreign language stimuli in the English language sessions, with experimenters being native Dutch speakers for the Dutch sessions or experimenters who do not speak Dutch for the English treatments. To control for related session differences and increase the efficiency of our estimations, we additionally included controls for session variation within the treatment group and within the control group (Models 9 and 12). We employed weighted contrast codes to be still able to interpret the coefficient estimated for the English treatment as the treatment effect. There are only statistically significant session effects across the treatment groups, but not across the control sessions. The former effect is due to a single session; excluding this session, no session effects remain $(\mathrm{F}(6,208)=0.71, \mathrm{p}=0.639)$, and our results are robust when excluding this session from the estimation (results available upon request). Since the effect of within-treatment group and within-control group variation on cooperation is rather small, we believe that experimenter and session characteristics that are not inherently related to the experimenter being Dutch or not Dutch did not severely bias our analyses.

\section{Discussion and Conclusion}

This study aims at further developing and testing a causal theory on how using a foreign language influences individuals' strategic decision-making in a global context. Reconsidering advances in psychological research on dual-process theories (Evans and Stanovich 2013; Stanovich 2009; Thompson 2009) and linking this to international business research (e.g., Tenzer et al. 2017; Volk et al. 2014), we demonstrate that information comprehension is an essential mediator for the effects of foreign language use on individuals' tendencies to cooperate. With our focus on language-induced variations in comprehension, we complement previous research on foreign language use (e.g., Costa et al. 2014a; Volk et al. 2014), which predominantly focuses on effects unrelated to variations in comprehension and empirically even ensures participants' comprehension. ${ }^{7}$ We call attention to previously neglected differences and interdependencies between the two sub-tasks leading

\footnotetext{
7 Specifically, our study leads to a refinement of conclusions suggested by Urbig et al. (2016), who investigated the effect of using English on cooperation among Dutch students. Their observation of foreign language use leading to less cooperative behavior, which we observe only for very proficient individuals with high levels of comprehension, is possibly a result of enforcing participants' comprehension. Our study suggests that this negative effect might turn positive for individuals less proficient in English who eventually suffer from reduced comprehension in foreign language contexts.
} 


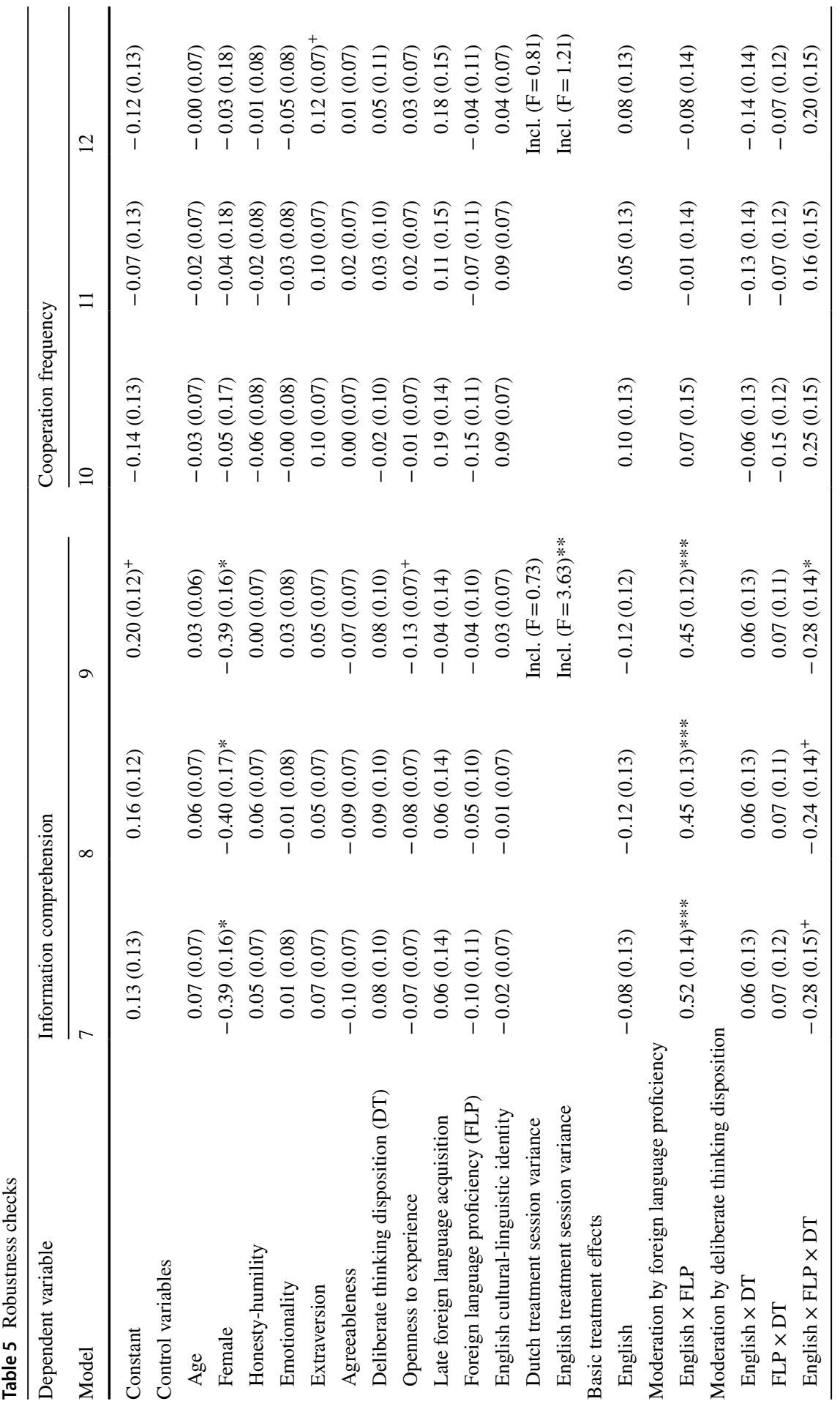




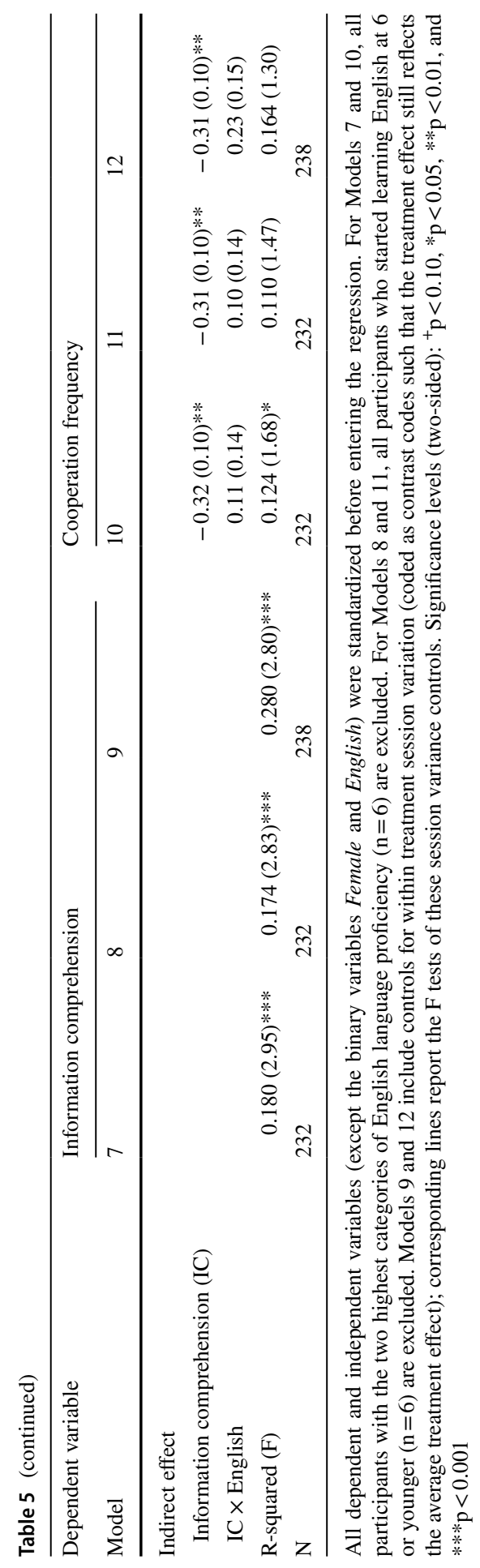


to a decision - that is, information processing and decision-making utilizing this information. This distinction, which mirrors similar distinctions in, among others, prospect theory (Kahneman and Tversky 1979), helps resolve previous debates on possibly opposing effects of foreign language use on thinking and decision-making (Hadjichristidis et al. 2017; Volk et al. 2014). Drawing on the role of personal characteristics in dual-process theories (Stanovich 2009) and recent research on language-related effects on decision-making (e.g., Urbig et al. 2016), we also demonstrate that foreign language proficiency and individual disposition toward deliberate thinking, as individual-level variables, moderate the relationship between foreign language use, as a contextual stimulus, and comprehension and cooperation behavior, respectively, as behavioral outcomes.

\subsection{Theoretical Contributions}

An increasing number of studies on foreign language effects are finding their way into economics and management research (e.g., Hicks et al. 2015; Ku and Zussman 2010; Mavisakalyan and Weber 2018; Micola et al. 2019; Neeley 2012, 2013; Neeley and Dumas 2016; Pan and Patel 2018; Sutter et al. 2018; Tenzer et al. 2017; Volk et al. 2014; Welch and Welch 2019), documenting far-reaching and fundamental effects of foreign language use on international business. However, we are still largely in the dark regarding the exact underlying causal mechanisms of why foreign language changes individuals' choices (e.g., Costa et al. 2014a; Hayakawa et al. 2016). Different theories spawn conflicting predictions. Specifically, Costa et al. (2014a) find that predictive ambiguity arises concerning the direction of language effects on decisionmaking. One stream of the literature suggests that using a nonnative language triggers less intuitive and heuristic reasoning, because using a foreign language reduces cognitive fluency, hence stimulating deliberate Type 2 thinking (e.g., Alter et al. 2007; Keysar et al. 2012). In contrast, other scholars propose that cognitive overload, resulting from straining mental resources due to using a foreign language, promotes intuitive Type 1 thinking (e.g., Volk et al. 2014). We extend this discussion by arguing that, indeed, seemingly conflicting predictions are consistent when the process linking foreign language use to behavior is conceptually split into language-related information processing, as one task, and decision-making that builds on the comprehended information, as another task (Hadjichristidis et al. 2017).

Increased working memory load, which could be triggered by using a foreign language, is primarily a characteristic of a reflective and deliberate type of information processing, enabling individuals to overcome potential threats from disfluency and low comprehension (Alter et al. 2007; Evans and Stanovich 2013; Thompson 2009). Hence, language-induced cognitive disfluency may attract mental resources to information processing, which likely distracts mental resources from other proximal, non-linguistic tasks competing for mental resources, such as making a decision. This shift in the allocation of cognitive resources might then lead to an intuitive and heuristic approach toward these latter tasks and a lower performance therein (e.g., Takano and Noda 1993, 1995). Hence, foreign language use may simultaneously lead to both more deliberate information processing and more heuristic 
decision-making. Note, however, that more heuristic decision-making only emerges if individuals increase their deliberation and employ more cognitive resources for information processing by sacrificing capacities that would otherwise (e.g., in the mother tongue) have been devoted to decision-making. If such competition for cognitive capacities leading to a cognitive overload is not present (e.g., in case of very simple and easy-to-comprehend settings; see, e.g., Keysar et al. 2012), the decisionmaking will not necessarily become more heuristic, but can, due to an alerting effect that attracts cognitive capacity not only to information processing but also to related decision-making, become even more deliberate (e.g., Keysar et al. 2012). ${ }^{8}$

Focusing on comprehension as the direct outcome of information processing and observing that individuals who are very proficient in the foreign language proficiency may even tend to report better comprehension in the foreign compared to native language treatments, our study strongly supports the accounts of Keysar et al. (2012) and Costa et al. (2014a) that using a foreign language stimulates more deliberate thinking. We believe that demonstrating the effect of comprehension is one of the most reliable available empirical tests because it targets the 'intended' consequence of the higher cognitive load triggered by using a foreign language-i.e., better comprehension.

Additionally, exploiting the novel conceptualization of our framework, this study identifies a previously marginalized mechanism through which foreign language use might affect individuals' decision-making. Prior related research mainly focuses on mechanisms that build on behavioral changes resulting from foreign language use activating more effortful information processing, which then either also triggers more deliberate decision-making due to an alerting effect that spills over from information processing to decision-making (Costa et al. 2014b; Keysar et al. 2012; Urbig et al. 2016) or more heuristic decision-making due to limited cognitive resources (Volk et al. 2014). However, effects stemming from foreign language reducing subjectively perceived comprehension are virtually unaddressed. Here, we propose that this mechanism might not only trigger more effortful information processing, but might also substantially alter individuals' decision-making. Specifically, if individuals who lack foreign language proficiency are unable to develop a sufficient understanding of the task context and report insufficient comprehension, their deliberate reasoning may fail to identify a satisfactory solution to the decision task (Ellsberg 1961). Consequently, individuals might display heuristic responses despite engaging in reflective and deliberate thinking,

\footnotetext{
${ }^{8}$ Interestingly, Volk et al.'s (2014) alternative attempt to account for the double-edged effect of foreign language use builds on foreign language proficiency moderating both the relationship between foreign language use and working memory load, as well as the relationship between working memory load and more heuristic decision-making. When hypothesizing about the base line effect for the second moderation, Volk and colleagues explicitly hypothesize that working memory load caused by foreign language use would stimulate heuristic decision-making. Thereby, they separate cognitive load due to information processing from cognitive load from other sources. Without this contextualization of the mediators' variation, they could not introduce foreign language proficiency as a related moderator and, thus, could not account for the double-edged effect. As demonstrated here, it is indeed this implicit distinction between working memory load needed for foreign language processing and working memory needed for other tasks, but not so much the double moderation by foreign language proficiency, which resolves the conflict between the two theorized effects.
} 
especially when deliberation cannot overcome threats to comprehension resulting from using a foreign language (Evans and Stanovich 2013; Thompson 2009).

\subsection{Practical Implications}

Joining an emerging stream of research studying decision-making through the lens of using a foreign language (e.g., Costa et al. 2014a; Geipel et al. 2016; Urbig et al. 2016; Volk et al. 2014; Welch and Welch 2019) and its application in international business research (Tenzer et al. 2017), this study highlights that firms that introduce English-only policies to cope with an increasingly linguistically diverse workforce, especially in international corporations, need to be aware of the subtle, but potentially profound, influences of nonnative speakers' use of English on their comprehension, decision-making, and behavior. The fundamental significance of the effect implies that it relates to the whole range of corporate domains including, among others, knowledge transfer (e.g., Welch and Welch 2008) and trust formation in multilingual team settings (Tenzer et al. 2014). Foreign language may alter the way individuals evaluate situations and make decisions. To the extent that foreign language use triggers less deliberate and more heuristic decision-making, decision quality may suffer.

Concerning the tendency to cooperate in foreign language settings (e.g., in multilingual teams), a particularly challenging effect emerges. While individuals might be more cooperative when communicating in a foreign language, such cooperation is likely to be fragile and unreliable as it rests primarily on an intuitive response. This intuitive response in the foreign language context may be overridden once, with more time available, individuals begin to assess the setting more deliberately. A subsequent breakdown of an already initiated cooperation may ultimately have an even more adverse impact on group dynamics than an immediate identification of controversy (as might have occurred if the native language had initially been used) would have had. Further, if initial cooperation decisions remain intact or are irreversible, team dynamics might suffer from regret related to previous decisions and resulting feelings of a lack of control. To address such dynamics, language and cross-cultural training targeted at overcoming the adverse effects associated with foreign language use might be more effective if they include an element of experiential learning about the subconscious effects of using a foreign language and, perhaps most critically, the potential inconsistencies that may result. Furthermore, more reflexivity-that is, a larger extent to which teams discuss task-related and process-related issues (Schippers et al. 2003) — might help people to become aware of adverse side effects of using a foreign language. Such increased awareness might, in turn, enable them to mitigate these adverse side effects better. More generally, however, reflexivity is a communicative process, which itself might be affected by using a foreign language. Hence, the positive effects of reflexivity on team dynamics themselves might be impaired when a foreign language is involved.

A related issue emanating from this study relates to separating the managerial tasks of information processing and decision-making in order to counter the harmful effects of using a foreign language. This separation could be achieved by 
appropriately designing structures and sequences of work meetings. Using a foreign language to communicate complex issues adds substantial cognitive load, which leaves less mental resources for decision processes. Leveraging information processing, by separating the processes of understanding and deciding-i.e., reducing the extent to which the two tasks compete for cognitive resources-could be achieved by presenting and discussing the available information and opinions in one meeting (i.e., a meeting focusing more on information comprehension). Summarizing considerations and a final decision could then be deferred to a separate second meeting (i.e., a meeting focusing more on the decision-making). The time between both meetings not only separates information processing from decision-making, thereby reducing the competition for mental resources, but also enables employees to engage in further information search, translation efforts or additional efforts to reinsure and increase one's confidence in having correctly understood the issues at hand. Such a meeting design might even enable firms to leverage the positive effects of using a foreign language. Foreign language use may trigger a more deliberate approach to meetings that participants otherwise would have approached in less deliberate ways. Well-targeted language and personal training along with suitable scheduling and design of important meetings held in a foreign language could potentially mitigate possible adverse effects of foreign language use, perhaps even turning threats into advantages. Developing practical tools and validating their hopefully positive effects is a promising avenue for relevant future research.

\subsection{Limitations and Future Research}

Like all studies, ours also has several limitations. We want to reflect briefly on some of the most salient, each offering a platform for promising future research. First, we focus on a specific combination of native (Dutch) and nonnative (English) languages. Given the growing importance of emerging countries and their languages, like China and Chinese (e.g., Maurais 2003), future research might explore whether our conclusions also hold for other countries and language combinations. These tests of generalization relate to the forceful plea to engage more in (extended) replications (Walker et al. 2019).

Second, for the experiment, we selected the price decision over a four-period nofeedback Bertrand duopoly as a relevant strategic business decision (see Akkermans et al. 2010). Individuals played against other individuals who participated in the same study program, but were otherwise anonymous. The specific set-up has the structure of a prisoner's dilemma game. In the literature, there is some discussion on whether the mindset associated with the prisoner's dilemma and the-often simplistic accompanying analyses - could be misleading for the normative analysis of both business and policy-making (Robèrt and Broman 2017; Shubik 1970). In particular, it has been suggested that using the prisoner's dilemma game might potentially promote an artificially hostile situation, which is not reflective of actual business situations, for example, negotiations. While we acknowledge the importance of these considerations, we do not derive any normative implications. Instead, we believe that the specific structure of the game allows us to identify the extent to 
which individuals may change their tendencies to cooperate depending on contextual factors (cf., Akkermans et al. 2010; Chen and Li 2005; Cox et al. 1991; Parkhe 1993; Zhang and Rajagopalan 2002).

Not allowing for feedback and playing against anonymous others implies that participants can base their decisions only on expectations about their peers, but not on their peers' actual characteristics. This independence of specific peers' characteristics increases the statistical power for tests of treatment effects and reduces potential confounding effects. However, the results must be interpreted very carefully concerning their generalization to more complex group dynamics (see the discussion by Zhou and Shi 2011). Individuals tend to become more cooperative once feedback is introduced or when an intense relationship exists and players have built up reputations (e.g., Akkermans et al. 2010; Andreoni and Miller 1993; Palfrey and Rosenthal 1994). Moreover, the analyses rest on the assumption that choosing the high price in a duopoly is perceived as akin to a cooperative strategy in a prisoner's dilemma. However, business people's perceptions of the 'game' that they are playing might differ from perceptions of individuals playing a prisoner dilemma in a laboratory context (Chattoe-Brown 2012; Robèrt and Broman 2017). Hence, future research should consider repeated decisions with feedback (Zhou and Shi 2011) and, possibly, even wholly different types of strategic business decisions, leaving behind the particular structure of a prisoner's dilemma situation.

Future research might also study more complex interactions, which allow for the establishment and the use of a meta-language and which might be affected by group and leadership dynamics as well as by subjective perceptions of these complex interactions. Meta-language refers to language that is used in order to describe and reflect upon communication in another language and is shown to contribute to enhanced understanding in other settings (e.g., Schleppegrell 2013). To the extent that the use of meta-language might leverage comprehension, it could also affect cooperation behavior as the ultimate outcome variable of interest. Superior comprehension might then facilitate more informed and more deliberate cooperation, which could result in less, but 'higher quality' cooperation. Hence, studies with more complex and repeated interaction could extend our work with its focus on individual decisions to also focus on emerging inter- and intra-organizational and group dynamics (e.g., Harzing and Feely 2008; Harzing et al. 2011; Neeley 2013; Neeley and Dumas 2016; Piekkari et al. 2005; Tenzer et al. 2014). In this context, future research might pay particular attention to transformational leadership, which has been demonstrated to be effective in dealing with team diversity, particularly with diverging nationalities (Kearney and Gebert 2009). It could be effective in dealing with linguistic diversity, too. Moreover, using ethnographic approaches, future research might further our understanding of how decision-makers perceive their environments, such that we can better model their decision structures-e.g., what decisions actually are perceived as reflecting more or less cooperative behavior (cf., Chattoe-Brown 2012).

Third, we identify a causal effect based on a rather homogenous business student sample. While the use of student samples is widespread in international business research that employs experiments to study the effects of using a foreign language (Fan and Harzing 2020) and is especially appropriate when fundamental 
effects are being studied (Bello et al. 2009), there might still be threats to the generalizability of results from students to a general population. Using business students, with their higher likelihood of being future business employees and managers, could be considered as striking a good balance between the benefits of a relatively homogeneous sample, but still being close to the business context. As discussed in the method section, student samples can be comparatively less susceptible to estimation biases, such as endogenous selection and unobserved heterogeneity, which is why they might not only be acceptable but, in some cases even a preferred population to study, primarily when the focal research issue addresses fundamental human decision making and does not require experiences in a specific domain (e.g., Bello et al. 2009; Bönte et al. 2016). When switching from student populations to field studies, future research is likely to need to adjust the employed research methods to leverage the unique advantages and adjust to the challenges related to field research. Researchers could build on our mediation framework to focus and simplify their field studies. They could either focus on the foreign language effect on comprehension and, thereby, put comprehension processes center stage, or focus on the comprehension effect on behavior and, thereby, broaden the scope to include more processes that may influence subjective perceptions of comprehension, others than foreign language use, such as, technological complexity. Concerning the methodological approaches, future research may seek to complement quantitative experimental research with other methods. Scholars may use qualitative interviews (e.g., Tenzer and Pudelko 2015) and employ mixed-methods designs (cf., Fan and Harzing 2020). Future field research could employ lab-in-the-field designs that facilitate the study of additional moderators related to the characteristics of employees' and managers' professional history, position in the company, and experience with cooperative decision-making in an international business context (e.g., multinational teams and international joint ventures). They may then use complementary in-depth interviews to let participants reflect upon their experiences in the experiment to shed more light on the qualitative processes underlying the foreign language effects.

Fourth, in our conceptual and empirical analysis, we focus on a single mechanism based on communication via written texts and focus solely on individuals' disposition to deliberate thinking as a moderator affecting the strength of the foreign language effect. Other mechanisms might be equally important at linking the use of a foreign language to comprehension and organizational behavior. We briefly reflect upon three of these. First, the behavioral change caused by using a foreign language might depend on the specific language and the associated culture (Akkermans et al. 2010). Different languages, embedded in distinct cultures, may trigger different processing algorithms and heuristics, thereby inducing not just the development of distinct sets of cognitions (e.g., Henderson 2005; Vaara et al. 2005), but also activating different values and norms (e.g., Bond and Yang 1982; Hong et al. 2000; Ralston et al. 1995). These effects could be highly relevant for comprehension in terms of sense-making (Brannen 2004), and for the weights attached to different goals and motives during decision-making processes. Analyses of related moderators, such as experience with foreign cultures (e.g., Akkermans et al. 2010) and country-specific social norms (e.g., Capraro and Cococcioni 2015), could fruitfully complement our 
study. Second, mental imagery is linked to using a foreign language (Hayakawa and Keysar 2018). The vividness of mental simulations is reduced in a foreign language context, and this reduction in the richness of mental imagery might at least partly be responsible for foreign language effects observed in prior research, favoring rational and utilitarian decisions over intuitive and emotionally charged ones. Hence, it might be interesting to explore whether intentionally increasing the vividness with which the task setting and consequences of decision options are described could mitigate a foreign language-induced reduction in the richness of mental imagery, thereby enhancing comprehension in foreign language contexts. Third, foreign language use might affect the emergence or breakdown of cooperation in second-language communication for other reasons that are not directly related to the comprehension-related mechanism, which is the focus of this study. A particular example relates to fault lines that may arise within multilingual teams based on differences in mother tongues, which might have adverse trust implications (e.g., Tenzer et al. 2014). Given the importance of trust and perceived trustworthiness for cooperative behavior in field settings, such effects might need to be considered in future field research, too. In sum, while making a relevant contribution concerning the role of comprehension for understanding the effect of foreign language use on strategic decision-making, many highly relevant and exciting issues emanate from and relate to our study, such that this study hopefully stimulates interesting future research on foreign language use in international management.

Acknowledgements Open Access funding provided by Projekt DEAL.

Open Access This article is licensed under a Creative Commons Attribution 4.0 International License, which permits use, sharing, adaptation, distribution and reproduction in any medium or format, as long as you give appropriate credit to the original author(s) and the source, provide a link to the Creative Commons licence, and indicate if changes were made. The images or other third party material in this article are included in the article's Creative Commons licence, unless indicated otherwise in a credit line to the material. If material is not included in the article's Creative Commons licence and your intended use is not permitted by statutory regulation or exceeds the permitted use, you will need to obtain permission directly from the copyright holder. To view a copy of this licence, visit http://creativecommons.org/licen ses/by/4.0/.

\section{References}

Akkermans, D., Harzing, A.-W., \& van Witteloostuijn, A. (2010). Cultural accommodation and language priming: Competitive versus cooperative behavior in a prisoner's dilemma game. Management International Review, 50(5), 559-584.

Alter, A. L., \& Oppenheimer, D. M. (2009). Uniting the tribes of fluency to form a metacognitive nation. Personality and Social Psychology Review, 13(3), 219-235.

Alter, A. L., Oppenheimer, D. M., Epley, N., \& Eyre, R. (2007). Overcoming intuition: Metacognitive difficulty activates analytic reasoning. Journal of Experimental Psychology: General, 136(4), 569-576.

Andreoni, J. (1995). Cooperation in public-goods experiments: Kindness or confusion? American Economic Review, 85(4), 891-904.

Andreoni, J., \& Miller, J. H. (1993). Rational cooperation in the finitely repeated prisoner's dilemma: Experimental evidence. Economic Journal, 103(418), 570-585. 
Bello, D., Leung, K., Radebaugh, L., Tung, R. L., \& vanWitteloostuijn, A. (2009). From the editors: Student samples in international business research. Journal of International Business Studies, 40(3), $361-364$.

Birdsong, D. (2006). Age and second language acquisition and processing: A selective overview. Language Learning, 56(S1), 9-49.

Bolton, G. E., \& Ockenfels, A. (2000). ERC: A theory of equity, reciprocity, and competition. American Economic Review, 90(1), 166-193.

Bond, M. H., \& Yang, K. S. (1982). Ethnic affirmation versus cross-cultural accommodation. The variable impact of questionnaire language on Chinese bilinguals from Hong Kong. Journal of CrossCultural Psychology, 13(2), 169-185.

Bönte, W., Procher, V. D., \& Urbig, D. (2016). Biology and selection into entrepreneurship: The relevance of prenatal testosterone exposure. Entrepreneurship Theory and Practice, 40(5), 1121-1148.

Boone, C., De Brabander, B., \& van Witteloostuijn, A. (1999). The impact of personality on behavior in five prisoner's dilemma games. Journal of Economic Psychology, 20(2), 343-377.

Bordia, S., \& Bordia, P. (2015). Employees' willingness to adopt a foreign functional language in multilingual organizations: The role of linguistic identity. Journal of International Business Studies, 46(4), 415-428.

Brannen, M. Y. (2004). When Mickey loses face: Recontextualization, semantic fit, and the semiotics of foreignness. Academy of Management Review, 29(4), 593-616.

Brannen, M. Y., Piekkari, R., \& Tietze, S. (2014). The multifaceted role of language in international business: Unpacking the forms, functions and features of a critical challenge to MNC theory and performance. Journal of International Business Studies, 45(5), 495-507.

Capraro, V., \& Cococcioni, G. (2015). Social setting, intuition and experience in laboratory experiments interact to shape cooperative decision-making. Proceedings of the Royal Society B: Biological Sciences, 282(1811), 20150237.

Chattoe-Brown, E. (2012). Combining ethnography and game theory using simulation: A critique and development of 'Can norms account for strategic interaction?' by S. Gezelius. Sociology, 46(2), 339-353.

Chen, X. P., \& Li, S. (2005). Cross-national differences in cooperative decision-making in mixed-motive business contexts. Journal of International Business Studies, 36(6), 622-636.

Chidlow, A., Plakoyiannaki, E., \& Welch, C. (2014). Translation in cross-language international business research: Beyond equivalence. Journal of International Business Studies, 45(5), 562-582.

Cohen, J., Cohen, P., West, S. G., \& Aiken, L. S. (2003). Applied multiple regression/correlation analysis for the behavioral sciences (3rd ed.). Mahwah, London: Lawrence Erlbaum Associates.

Cornelissen, G., Dewitte, S., \& Warlop, L. (2011). Are social value orientations expressed automatically? Decision making in the dictator game. Personality and Social Psychology Bulletin, 37(8), 1080-1090.

Costa, A., Foucart, A., Arnon, I., Aparici, M., \& Apesteguia, J. (2014a). "Piensa' twice: On the foreign language effect in decision making. Cognition, 130, 236-254.

Costa, A., Foucart, A., Hayakawa, S., Aparici, M., Apesteguia, J., Heafner, J., et al. (2014b). Your morals depend on language. PLoS One, 9(4), e94842.

Cox, T. H., Lobel, S. A., \& McLeod, P. L. (1991). Effects of ethnic group cultural differences on cooperative and competitive behavior on a group task. Academy of Management Journal, 34(4), 827-847.

Crystal, D. (2003). English as a global language (2nd ed.). Cambridge: Cambridge University Press.

Dalal, D. K., \& Zickar, M. J. (2012). Some common myths about centering predictor variables in moderated multiple regression and polynomial regression. Organizational Research Methods, 15(3), 339-362.

Duffy, S., \& Smith, J. (2014). Cognitive load in the multi-player prisoner's dilemma game: Are there brains in games? Journal of Behavioral and Experimental Economics, 51, 47-56.

Elfenbein, D. W., Knott, A. M., \& Croson, R. (2017). Equity stakes and exit: An experimental approach to decomposing exit delay. Strategic Management Journal, 38(2), 278-299.

Ellsberg, D. (1961). Risk, ambiguity, and the savage axioms. Quarterly Journal of Economics, 75(4), 643-669.

Evans, J. S. B., \& Stanovich, K. E. (2013). Dual-process theories of higher cognition: Advancing the debate. Perspectives on Psychological Science, 8(3), 223-241.

Fan, S. X., \& Harzing, A. W. (2020). Moving beyond the baseline: Exploring the potential of experiments in language research. In S. Horn et al. (Eds.), Understanding multilingual workplaces: Methodological, empirical and pedagogic perspectives. Abbington: Routledge. 
Feely, A. J., \& Harzing, A.-W. (2003). Language management in multinational companies. Cross Cultural Management: An International Journal, 10(2), 37-52.

Fehr, E., \& Schmidt, K. M. (1999). A theory of fairness, competition and cooperation. Quarterly Journal of Economics, 114(3), 817-868.

Fox, C. R., \& Tversky, A. (1995). Ambiguity aversion and comparative ignorance. Quarterly Journal of Economics, 110(3), 585-603.

Francis, W. S., \& Gutiérrez, M. (2012). Bilingual recognition memory: Stronger performance but weaker levels-of-processing effects in the less fluent language. Memory \& Cognition, 40(3), 496-503.

Frederick, S. (2005). Cognitive reflection and decision making. Journal of Economic Perspectives, 19(4), $25-42$.

Frederiksson, R., Barner-Rasmussen, W., \& Piekkari, R. (2006). The multinational corporation as a multilingual organization: The notion of a common corporate language. Corporate Communications: An International Journal, 11(4), 406-423.

Geipel, J., Hadjichristidis, C., \& Surian, L. (2016). Foreign language affects the contribution of intentions and outcomes to moral judgment. Cognition, 154, 34-39.

Gerber, A. S., \& Green, D. P. (2012). Field experiments: Design, analysis, and interpretation. New York: WW Norton.

Gumperz, J. J., \& Gumperz, J. C. (1996). Introduction: Language and the communication of social identity. In J. J. Gumperz (Ed.), Language and social identity (pp. 1-21). New York: Cambridge University Press.

Hadjichristidis, C., Geipel, J., \& Surian, L. (2017). How foreign language affects decisions: Rethinking the brain-drain model. Journal of International Business Studies, 48(5), 645-651.

Harzing, A.-W., \& Feely, A. J. (2008). The language barrier and its implications for HQ-subsidiary relationships. Cross-Cultural Management: An International Journal, 15(1), 49-61.

Harzing, A.-W., Köster, K., \& Magner, U. (2011). Babel in business: The language barrier and its solutions in the HQ-subsidiary relationship. Journal of World Business, 46(3), 279-287.

Hayakawa, S., \& Keysar, B. (2018). Using a foreign language reduces mental imagery. Cognition, 173, $8-15$.

Hayakawa, S., Costa, A., Foucart, A., \& Keysar, B. (2016). Using a foreign language changes our choices. Trends in Cognitive Sciences, 20(11), 791-793.

Hayakawa, S., Tannenbaum, D., Costa, A., Corey, J. D., \& Keysar, B. (2017). Thinking more or feeling less? Explaining the foreign-language effect on moral judgment. Psychological science, 28(10), 1387-1397.

Henderson, J. K. (2005). Language diversity in international management teams. International Studies of Management and Organization, 35(1), 66-82.

Hernandez, A. R., \& Li, P. (2007). Age of acquisition: Its neural and computational mechanisms. Psychological Bulletin, 133(4), 638-650.

Hicks, D. L., Santacreu-Vasut, E., \& Shoham, A. (2015). Does mother tongue make for women's work? Linguistics, household labor, and gender identity. Journal of Economic Behavior and Organization, 110, 19-44.

Hong, Y.-Y., Morris, M. W., Chiu, C.-Y., \& Benet-Martínez, V. (2000). Multicultural minds: A dynamic constructivist approach to culture and cognition. American Psychologist, 55(7), 709-720.

Jones, R. J., \& George, J. M. (1998). The experience and evolution of trust: Implications for cooperation and teamwork. Academy of Management Review, 23(3), 531-546.

Just, M. A., \& Carpenter, P. A. (1992). A capacity theory of comprehension: Individual differences in working memory. Psychological Review, 99(1), 122-149.

Kahneman, D. (2011). Thinking, fast and slow. New York: Macmillan.

Kahneman, D., \& Tversky, A. (1979). Prospect theory: An analysis of decision under risk. Econometrica, 47(2), 363-391.

Kearney, E., \& Gebert, D. (2009). Managing diversity and enhancing team outcomes: The promise of transformational leadership. Journal of Applied Psychology, 94(1), 77-89.

Keysar, B., Hayakawa, S. L., \& An, S. G. (2012). The foreign-language effect: Thinking in a foreign tongue reduces decision biases. Psychological Science, 23(6), 661-668.

Ku, H., \& Zussman, A. (2010). Lingua franca: The role of english in international trade. Journal of Economic Behavior and Organization, 75(2), 250-260.

Lee, K., \& Ashton, M. C. (2004). Psychometric properties of the HEXACO personality inventory. Multivariate Behavioral Research, 39(2), 329-358. 
Maurais, J. (2003). Towards a new linguistic world order. In J. Maurais \& M. A. Morris (Eds.), Languages in a globalising world (pp. 13-36). Cambridge: Cambridge University Press.

Mavisakalyan, A., \& Weber, C. (2018). Linguistic structures and economic outcomes. Journal of Economic Surveys, 32(3), 916-939.

McCrae, R. R., \& John, O. P. (1992). An introduction to the five-factor model and its applications. Journal of Personality, 60(2), 175-215.

McGrath, R. G. (2001). Exploratory learning, innovative capacity, and managerial oversight. Academy of Management Journal, 44(1), 118-131.

Micola, A. R., Fenoll, A. A., Banal-Estañol, A., \& Bris, A. (2019). TV or not TV? The impact of subtitling on English skills. Journal of Economic Behavior and Organization, 158, 487-499.

Mosakowski, E. (1997). Strategy making under causal ambiguity: Conceptual issues and empirical evidence. Organizational Science, 8(4), 414-442.

Neeley, T. B. (2012). Global business speaks English: Why you need a language strategy now. Harvard Business Review, 90(5), 116-124.

Neeley, T. B. (2013). Language matters: Status loss and achieved status distinctions in global organizations. Organizational Science, 24(2), 476-497.

Neeley, T., \& Dumas, T. L. (2016). Unearned status gain: Evidence from a global language mandate. Academy of Management Journal, 59(1), 14-43.

Oganian, Y., Korn, C. W., \& Heekeren, H. R. (2016). Language switching-but not foreign language use per se-Reduces the framing effect. Journal of Experimental Psychology: Learning, Memory, and Cognition, 42(1), 140-148.

Palfrey, T. R., \& Rosenthal, H. (1994). Repeated play, cooperation and coordination: An experimental study. The Review of Economic Studies, 61(3), 545-565.

Pan, P., \& Patel, C. (2018). The influence of native versus foreign language on Chinese subjects' aggressive financial reporting judgments. Journal of Business Ethics, 150(3), 863-878.

Parkhe, A. (1993). Strategic alliance structuring: A game theoretic and transaction cost examination of interfirm cooperation. Academy of Management Journal, 36(4), 794-829.

Peysakhovich, A., \& Rand, D. G. (2016). Habits of virtue: Creating norms of cooperation and defection in the laboratory. Management Science, 62(3), 631-647.

Piekkari, R., Vaara, E., Tienari, J., \& Säntti, R. (2005). Integration or disintegration? Human resource implications of the common corporate language decision in a cross-border merger. The International Journal of Human Resource Management, 16(3), 330-344.

Preacher, K. J., Rucker, D. D., \& Hayes, A. F. (2007). Addressing moderated mediation hypotheses: Theory, methods, and prescriptions. Multivariate Behavioral Research, 42(1), 185-227.

Ralston, D. A., Cunniff, M. K., \& Gustafson, D. J. (1995). Cultural accommodation: The effect of language on the responses of bilingual Hong Kong Chinese managers. Journal of Cross-Cultural Psychology, 26(6), 714-727.

Rand, D. G. (2016). Cooperation, fast and slow: Meta-analytic evidence for a theory of social heuristics and self-interested deliberation. Psychological Science, 27(9), 1192-1206.

Rand, D. G. (2019). Intuition, deliberation, and cooperation: Further meta-analytic evidence from 91 experiments on pure cooperation. Available at SSRN 3390018.

Rand, D. G., Greene, J. D., \& Nowak, M. A. (2012). Spontaneous giving and calculated greed. Nature, 489, 427-430.

Rand, D. G., Peysakhovich, A., Kraft-Todd, G. T., Newman, G. E., Wurzbacher, O., Nowak, M. A., et al. (2014). Social heuristics shape intuitive cooperation. Nature Communications, 5, 3677.

Robèrt, K. H., \& Broman, G. (2017). Prisoners' dilemma misleads business and policy making. Journal of Cleaner Production, 140(Part 1), 10-16.

Schippers, M. C., Den Hartog, D. N., Koopman, P. L., \& Wienk, J. A. (2003). Diversity and team outcomes: The moderating effects of outcome outcome interdependence and group longevity and the mediating effect of reflexivity. Journal of Organizational Behavior, 24(6), 779-802.

Schleppegrell, M. J. (2013). The role of metalanguage in supporting academic language development. Language Learning, 63(1), 153-170.

Shamir, B., \& Kark, R. (2004). A single-item graphic scale for the measurement of organizational identification. Journal of Occupational and Organizational Psychology, 77(1), 115-123.

Shubik, M. (1970). Game theory, behavior, and the paradox of the prisoner's dilemma: Three solutions. Journal of Conflict Resolution, 14(2), 181-193.

Slovic, P., Finucane, M. L., Peters, E., \& MacGregor, D. G. (2007). The affect heuristic. European Journal of Operational Research, 177(3), 1333-1352. 
Stanovich, K. E. (2009). Distinguishing the reflective, algorithmic and autonomous minds: Is it time for a tri-process theory? In J. S. B. Evans \& K. Frankish (Eds.), In two minds: Dual processes and beyond (pp. 55-88). Oxford: Oxford University Press.

Sullivan, J. J. (1988). Three roles of language in motivation theory. Academy of Management Review, $13(1), 104-115$.

Sutter, M., Angerer, S., Glätzle-Rützler, D., \& Lergetporer, P. (2018). Language group differences in time preferences: Evidence from primary school children in a bilingual city. European Economic Review, 106(C), 21-34.

Takano, Y., \& Noda, A. (1993). A temporary decline of thinking ability during foreign language processing. Journal of Cross-Cultural Psychology, 24(4), 445-462.

Takano, Y., \& Noda, A. (1995). Interlanguage dissimilarity enhances the decline of thinking ability during foreign language processing. Language Learning, 45(4), 657-681.

Tenzer, H., \& Pudelko, M. (2015). Leading across language barriers: Managing language-induced emotions in multinational teams. The Leadership Quarterly, 26(4), 606-625.

Tenzer, H., Pudelko, M., \& Harzing, A.-W. (2014). The impact of language barriers on trust formation in multinational teams. Journal of International Business Studies, 45(5), 508-535.

Tenzer, H., Terjesen, S., \& Harzing, A. W. (2017). Language in international business: A review and agenda for future research. Management International Review, 57(6), 815-854.

Thompson, V. A. (2009). Dual-process theories: A metacognitive perspective. In J. S. B. Evans \& K. Frankish (Eds.), In two minds: Dual processes and beyond (pp. 171-196). Oxford: Oxford University Press.

Tsai, W. (2002). Social structure of 'coopetition' within a multiunit organization: Coordination, competition, and intraorganizational knowledge sharing. Organization Science, 13(2), 179-190.

Tversky, A., \& Kahneman, D. (1992). Advances in prospect theory: Cumulative representation of uncertainty. Journal of Risk and Uncertainty, 5(4), 297-323.

Urbig, D., Terjesen, S., Procher, V., Muehlfeld, K., \& van Witteloostuijn, A. (2016). Come on and take a free ride: Contributing to public goods in native and foreign language settings. Academy of Management Learning and Education, 15(2), 268-286.

Vaara, E., Tienari, J., Piekkari, R., \& Säntti, R. (2005). Language and the circuits of power in a merging multinational corporation. Journal of Management Studies, 42(3), 595-623.

Vives, M. L., Aparici, M., \& Costa, A. (2018). The limits of the foreign language effect on decision-making: The case of the outcome bias and the representativeness heuristic. PloS one, 13(9), e0203528.

van Witteloostuijn, A. (2015). Toward experimental international business: Unraveling fundamental causal linkages. Cross-Cultural and Strategic Management, 22(4), 530-544.

Volk, S., Köhler, T., \& Pudelko, M. (2014). Brain drain: The cognitive neuroscience of foreign language processing in multinational corporations. Journal of International Business Studies, 45(7), $862-885$.

Walker, R. M., Brewer, G. A., Lee, M. J., Petrovsky, N., \& van Witteloostuijn, A. (2019). Best practice recommendations for replicating experiments in public administration. Journal of Public Administration Research and Theory, 29(4), 609-626.

Weber, J. M., \& Murnighan, J. K. (2008). Suckers or saviors? Consistent contributors in social dilemmas. Journal of Personality and Social Psychology, 95(6), 1340-1353.

Welch, D. E., \& Welch, L. S. (2008). The importance of language in international knowledge transfer. Management International Review, 48(3), 339-360.

Welch, D. E., \& Welch, L. S. (2019). Coping with multilingualism: Internationalization and the evolution of language strategy. Global Strategy Journal, 9(4), 618-639.

Williams, D. W., Wood, M. S., Mitchell, J. R., \& Urbig, D. (2019). Applying experimental methods to advance entrepreneurship research: On the need for and publication of experiments. Journal of Business Venturing, 34(2), 215-223.

Zaki, J., \& Mitchell, J. P. (2013). Intuitive prosociality. Current Directions in Psychological Science, 22(6), 466-470.

Zellmer-Bruhn, M., Caligiuri, P., \& Thomas, D. C. (2016). From the editors: Experimental designs in international business research. Journal of International Business Studies, 47(4), 399-407.

Zeng, M., \& Chen, X. P. (2003). Achieving cooperation in multiparty alliances: A social dilemma approach to partnership management. Academy of Management Review, 28(4), 587-605.

Zhang, Y., \& Rajagopalan, N. (2002). Inter-partner credible threat in international joint ventures: An infinitely repeated prisoner's dilemma model. Journal of International Business Studies, 33(3), $457-478$. 
Zhou, W., \& Shi, X. (2011). Culture in groups and teams: A review of three decades of research. International Journal of Cross-Cultural Management, 4(1), 5-34.

Publisher's Note Springer Nature remains neutral with regard to jurisdictional claims in published maps and institutional affiliations.

\section{Affiliations}

\section{Diemo Urbig ${ }^{1,2} \mathbb{D} \cdot$ Katrin Muehlfeld ${ }^{3,4} \cdot$ Vivien D. Procher ${ }^{5,6}$ (D) Arjen van Witteloostuijn ${ }^{7,8}$}

1 Institute of Business and Economics, Brandenburg University of Technology CottbusSenftenberg, Cottbus, Germany

2 Institute for Development Strategies, School of Public and Environmental Affairs, Indiana University, Bloomington, USA

3 Department of Business Administration, Trier University, Trier, Germany

4 Utrecht University School of Economics, Utrecht University, Utrecht, The Netherlands

5 Grenoble Ecole de Management, Univ Grenoble Alpes ComUE, Grenoble, France

6 RWI-Leibniz-Institut für Wirtschaftsforschung, Essen, Germany

7 School of Business and Economics, Vrije Universiteit Amsterdam, Amsterdam, The Netherlands

8 Antwerp Management School, Antwerp University, Antwerp, Belgium 